\title{
Existence and Stability of Overconsumption Equilibria*
}

\author{
Gregory Ponthiere ${ }^{\dagger}$
}

July 27, 2010

\begin{abstract}
Growth models with endogenous mortality assume generally that life expectancy is increasing with output per capita and, thus, with individual consumption, whatever its level is. However, empirical evidence supports a U-shaped relationship between consumption and mortality, implying that the monotonicity of that relation is local but not global. This paper develops a two-period OLG model where life expectancy is a non-monotone function of consumption, and where agents form myopic anticipations about life expectancy. The existence, uniqueness and stability of steady-state equilibria are studied. It is shown that overconsumption equilibria - i.e. equilibria at which consumption exceeds the level maximizing life expectancy - exist in highly productive economies with a low impatience. We identify also conditions under which there exist long-run cycles in output and longevity around overconsumption equilibria.

Keywords: longevity, mortality, growth, overconsumption, OLG model.

JEL codes: E13, E21, I12.
\end{abstract}

\section{Introduction}

In the recent decades, economists have paid particular attention to the relationship between economic development and survival conditions. That relationship was studied both at the empirical and theoretical levels.

On the empirical side, studies by Preston $(1975,1980)$, Anand and Ravallion (1993), Kelley and Schmidt (1994, 1995) and Pritchett and Summers (1996) report an increasing and concave relationship between output and longevity. More recently, Boucekkine et al (2009) provide a nonparametric inference of the relationship between life expectancy and economic growth on the basis of a dataset including 18 countries over 1820-2005. The relation between the economic growth rate and life expectancy is shown to be non-monotone, confirming the existence of a non-linear relation between the levels of output and longevity.

On the theoretical side, several models examined the impact of survival conditions - taken as exogenous - on capital accumulation and growth. Such models include Ehrlich and Lui (1991), de la Croix and Licandro (1999) and

\footnotetext{
*The author would like to thank Gabrielle Demange, Fabrice Etilé, Pierre-Yves Geoffard and two anonymous referees for helpful comments on this paper.

${ }^{\dagger}$ Ecole Normale Superieure and Paris School of Economics (PSE). Address: ENS, boulevard Jourdan, 48, building B, 75014 Paris, France. Contact: gregory.ponthiere@ens.fr
} 
Boucekkine et al (2002). The latter model shows that a higher life expectancy raises the human capital stock through a rise in the number of persons, and favours investment in schooling through a horizon effect, but leads also to the ageing of the stock of human capital, so that the relation between longevity and human capital is non-monotone. Under low longevity levels, economic growth and longevity are positively related, but not under high longevity levels. ${ }^{1}$

While those models assume exogenous longevity, other papers, such as Blackburn and Cipriani (2002) and Chakraborty (2004), study the output / longevity relationship while modelling survival conditions as an output of the economy. ${ }^{2}$ A major common feature of those frameworks is the modelling of the twodirectional relation between economic growth and survival conditions. Longevity affects accumulation decisions (e.g. savings, schooling) through a horizon effect, but there exists also a feedback effect, from economic development to survival conditions. In general, that feedback mechanism takes a simple, monotone form: economic growth is assumed to raise longevity through a survival function that is increasing in (human or physical) capital (through public and/or private health spending). Hence, in those models, the endogenization of survival conditions does not affect the global shape of the relationship between development and survival conditions. A higher life expectancy leads to a higher output, and, thanks to the monotone feedback effect, life expectancy grows "hand-in-hand" with all economic variables, i.e. output, consumption, savings. ${ }^{3}$

The goal of this paper is to complement that theoretical literature, by paying a particular attention to the precise form of the feedback mechanism from economic activity to human longevity. Assuming a monotone influence of output on survival conditions is analytically convenient, since a survival function monotone in one (or several) input(s) is the simplest way to endogenize longevity. However, simplicity has also its costs, and modelling the production of life-years exactly as the production of goods may involve a too strong reduction. Actually, those two production processes - i.e. goods and life-years - may differ qualitatively. Whereas the former production process is certainly monotone in its inputs, it is not obvious at all that the latter production process is also a monotone one. Let us thus now examine in more details the plausibility of a monotone feedback effect from economic development to survival conditions.

A first way to test for the plausibility of a monotone feedback effect is to examine whether it is really the case that all economic variable evolve handin-hand with life expectancy, as predicted by existing models. Actually, one can question the monotonicity postulate on the basis of one of its corollaries, concerning the longevity / consumption relation. Existing models predict that longevity must be increasing monotonically with consumption. The problem is that this is not compatible with the data. If one adopts a cross-sectional perspective and plots the levels of consumption and life expectancy across countries, it is easy to see that the relationship between the two variables is far from monotone. As shown by the tendency curve drawn on Figure 1, life expectancy at birth is increasing with consumption per capita only up to some level of consumption, but, beyond that level, life expectancy is declining in consumption. ${ }^{4}$

\footnotetext{
${ }^{1}$ On the empirical assessment of that model, see Boucekkine et al $(2003,2009)$.

${ }^{2}$ See also Bhattacharya and Qiao (2005), Cervellati and Sunde (2005), Chakraborty and Das (2005), Galor and Moav (2005), Zhang et al (2006) and de la Croix and Licandro (2007).

${ }^{3}$ One exception is Jouvet et al (2010), where production-related pollution reduces longevity.

${ }^{4}$ The countries of Figure 1 are: Australia, Austria, Belgium, Canada, Czech Republic, Fin-
} 


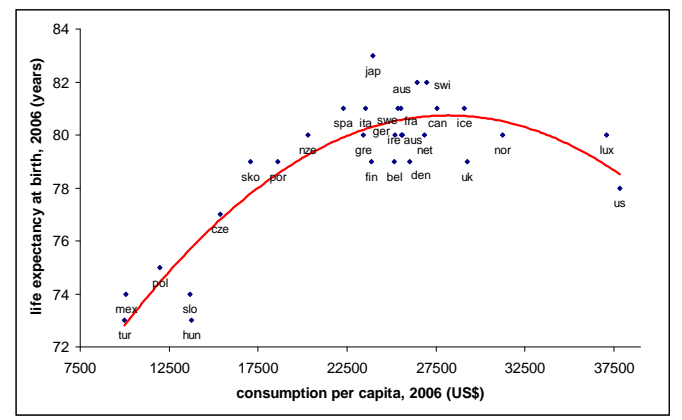

Figure 1: Consumption per head and life expectancy at birth, 2006.

Obviously, Figure 1 is not a proof of the non-monotonicity of the relation under study: the observed relationship may actually hide the influence of omitted variables correlated with consumption and influencing life expectancy in a nonmonotone way. Nevertheless, Figure 1 has a strong corollary for the modelling of longevity in models where survival functions have a single input. Clearly, Figure 1 suggests that if one wants, on the grounds of analytical tractability, to keep a survival function with a unique input (either consumption or another variable correlated with it), the monotonicity postulate is hard to justify. ${ }^{5}$

Beyond the incapacity of monotone survival functions to fit aggregate data, the monotonicity postulate can also be questionned in the light of the large epidemiological literature on the negative effects of overconsumption on survival. ${ }^{6}$ Epidemiological studies emphasized a non-monotone relationship between the corpulence (measured by the body mass index - BMI) and mortality risks. For instance, Adams et al (2006)'s study, which focused on a sample of more than 61,000 subjects, estimated that overweighted persons exhibit a risk of death that is between 20 and $40 \%$ higher than the one faced by normal persons. ${ }^{7}$ Under the - quite mild - postulate of a link between food consumption and corpulence, it is straigthfoward to deduce that more consumption is not necessarily better for health and survival. Although more consumption is good for health up to some level, excessive consumption becomes bad for health and survival.

The non-monotone relation between corpulence and survival prospects is best described by Waaler (1984)'s U-shaped curves in the (BMI, mortality) space (see Figure 2). ${ }^{8}$ Those curves were interpreted by Fogel (1994) from an intertemporal perspective. ${ }^{9}$ During the longest part of history, populations have been undernourrished, so that the average BMI has remained quite low, and

land, France, Germany, Greece, Hungary, Iceland, Ireland, Italy, Japan, Luxemburg, Mexico, New Zealand, Netherlands, Norway, Poland, Portugal, Slovakia, South Korea, Spain, Sweden, Switzerland, Turkey, the United Kingdom, and the United States. Consumption statistics (in US\$ with current PPPs) are from the OECD (2009). Period life expectancy statistics (average for both sexes) are from the World Health Organization (2009).

${ }^{5}$ Note that the picture would be roughly the same if we plotted life expectancy and income.

${ }^{6}$ See Solomon and Manson (1997), Bender et al (1998), Fontaine et al (2003), Breeze et al (2005) and Adams et al (2006).

${ }^{7}$ For obese persons, the risk of death is two and three times higher than the norm.

${ }^{8}$ Those curves have been reproduced from Fogel (1994, p. 376).

${ }^{9}$ On the links between survival, BMI and food consumption, see also Fogel (2004). 
mortality quite large (left of the graph). However, there has been a rise in the quantity of calories taken by agents since the 18th century, and so populations have been moving towards a lower mortality (right of the graph). Moreover, the rightest part of Waaler's curves shows the negative effects of overconsumption on survival prospects highlighted by epidemiological studies.

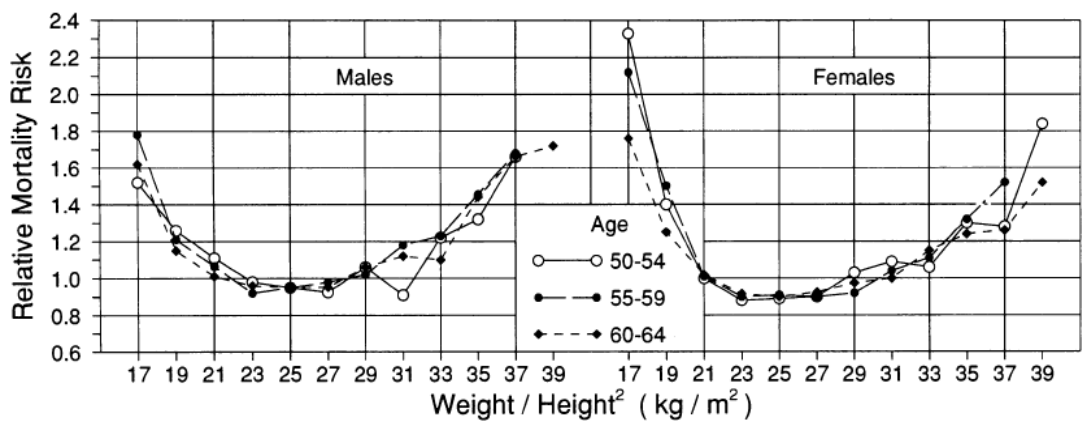

Figure 2: BMI and mortality among Norwegian adults aged 50-64 (1963-1979)

The right part of Waaler's curves and, more generally, the voluminous epidemiological literature on the effects of overconsumption have recently attracted the attention of economists in what is called the "economics of obesity". ${ }^{10}$ That emerging field has, among other things, studied the economic determinants of obesity, such as the secular fall of food prices induced by technological progress (see Lakdawalla and Philipson, 2002). It has also questionned the capacity of agents to anticipate the effects of their consumption choices on future health, and proposed several behavioural theories aimed at explaining obesity. ${ }^{11}$

Despite the expanding literature on overconsumption, there has been so far no attempt, within growth theory, to account for the non-monotone relationship between consumption and survival prospects. Existing models made survival depend monotonically on a variable correlated positively with consumption, e.g. (physical or human) capital. But such a simplification may not be benign for the dynamics of output and mortality. The monotonicity assumption, although analytically convenient, may truncate the long-run dynamics of the economy.

The goal of this paper is precisely to study the dynamics of longevity and production in an economy à la Fogel, where survival depends on consumption in a non-monotone manner (as shown by Waaler's curves). For that purpose, we develop a two-period OLG model with physical capital accumulation and endogenous survival, which is largely in line with Chakraborty (2004). However, a major difference with respect to Chakraborty's model is that, in our economy, life expectancy is increasing with first-period consumption up to some "healthy" consumption level, but starts declining for higher consumption levels. As a consequence of this, life expectancy is no longer increasing monotonically with physical capital (unlike in Chakraborty's model). ${ }^{12}$

\footnotetext{
${ }^{10}$ See the survey of Philipson and Posner (2008).

${ }^{11}$ See, for instance, Cutler et al (2003).

12 Our modelling makes the production of the good and of life-years differ qualitatively: for the former, a larger input (capital) leads always to a larger output, whereas, for the latter, a larger input (consumption) leads to a higher or a lower output, depending on the input level.
} 
In economies with a large production capacity, the long-run equilibrium may involve some overconsumption, i.e. a consumption exceeding the level that maximizes survival prospects. That possibility was excluded in existing models, which relied on the "more is always better" postulate. In contrast, this paper pays a particular attention to the conditions guaranteeing the existence of overconsumption equilibria. A strong emphasis will also be laid on the stability of those equilibria. Can there be stable overconsumption equilibria? Or should we expect non-converging cycles to occur? This paper proposes to cast a new light on the existence and stability of overconsumption equilibria.

For that purpose, we will make here several simplifying assumptions, which allow us to focus on the problem at stake. First, we will remain, for simplicity, in a one-good economy, as in almost all OLG models with endogenous survival. Second, we will keep a survival function that depends on a single input (consumption) and ignore health spendings. We do so to be in line with Fogel's theory of long-run development: from a long-run perspective, consumption constitutes the most natural input to enter the survival function. Third, we assume no heterogeneity within cohorts, but only between cohorts. This is another simplification, as cohorts are, in reality, composed of agents differing in various dimensions affecting survival. ${ }^{13}$ Fourth, we assume that agents form adaptive anticipations regarding their survival prospects. Thus, agents make their savings decision on the basis of the prevailing period life-tables, which are taken as given. That assumption makes sense, but remains a simplification, which we will have to keep in mind when interpreting our results. ${ }^{14}$

Anticipating our results, we show that stationary overconsumption equilibria - i.e. equilibria at which consumption exceeds the level maximizing life expectancy - exist in highly productive economies with low impatience. Thus, contrary to common sense, overconsumption is, from a dynamic perspective, the outcome of patience rather than impatience. Moreover, stability analysis highlights formal conditions under which there exist non-converging cycles in output and longevity around overconsumption equilibria. Thus the mere possibility of (health-damaging) overconsumption constitutes an additional source of cycles, which we relate to the existing literature on economic and demographic cycles.

The rest of the paper is organized as follows. The model is presented in Section 2. Section 3 examines the existence, uniqueness and stability of steadystate equilibria. Section 4 illustrates the dynamics of production and longevity on the basis of numerical simulations. Section 5 discusses our results in the light of the literature on economic and demographic cycles. Section 6 concludes.

\section{The model}

Let us consider a two-period OLG model. For simplicity, we assume that reproduction is monosexual, and that each person gives birth to exactly one child.

The first period is a period of young adulthood, during which the adult gives birth to a child, supplies his labour inelastically and saves some resources for the old age. The second period is a period of retirement.

\footnotetext{
${ }^{13}$ See Galor and Moav (2005) and Ponthiere (2010).

${ }^{14}$ In real life, individuals, although ignorant of Waaler's curves, may still be aware of some - but not all - of the effects at work, and may extrapolate survival chances accordingly.
} 
Survival conditions Only a fraction $\pi_{t+1}$ of a cohort born at time $t$ reaches the retirement age. ${ }^{15}$ Following the empirical literature surveyed in the introduction, we assume that the fraction $\pi_{t+1}$ depends on the consumption when being young $c_{t}$ in a non-monotone way. This amounts to assuming that there exists a level of consumption $c^{*}$ that yields the maximum survival probability. Any departure from $c^{*}$, either from below or from above, generates a lower survival probability. ${ }^{16}$

For simplicity, the probability of survival to the old age is assumed to be determined by the following survival function

$$
\pi_{t+1}=\frac{1}{1+\eta\left(c^{*}-c_{t}\right)^{2}}
$$

where $c^{*}>0$ is the "healthy" consumption level, that is, the consumption level that yields the maximum life expectancy. The parameter $\eta \geq 0$ captures the impact of consumption on survival prospects. Note that $\lim _{c_{t} \rightarrow 0} \pi_{t+1}=$ $\frac{1}{1+\eta c^{* 2}} \equiv \bar{\pi}$, which is close to zero when the healthy consumption level $c^{*}$ is high. Moreover, we have that $\lim _{c_{t} \rightarrow c^{*}} \pi_{t+1}=1$ and $\lim _{c_{t} \rightarrow \infty} \pi_{t+1}=0$.

Figure 3 illustrates the relationship between $\pi_{t+1}$ and $c_{t}$. When consumption is inferior to the healthy consumption level $c^{*}$, a higher consumption raises survival prospects. On the contrary, if $c_{t}>c^{*}$, the opposite prevails: a lower consumption would raise life expectancy.

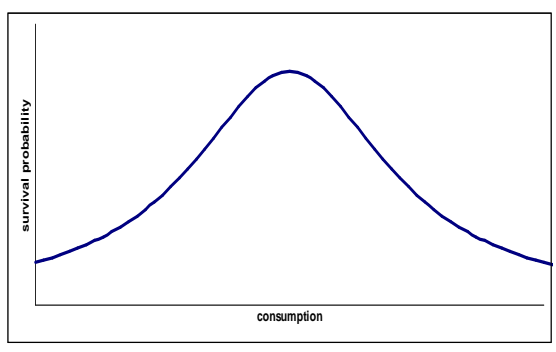

Figure 3: $\pi_{t+1}$ as a function of $c_{t}$

One can interpret Figure 3 as reflecting, within our simple one-good economy, the U-shaped curves in the (BMI, mortality) space discussed by Fogel (1994). Actually, if food consumption and the BMI are positively related, it is quite natural to deduce, from a U-shaped relation between BMI and mortality, an inverted-U shaped relation between consumption and life expectancy. ${ }^{17}$ Note that our survival function is convex at low levels of consumption, and, then, concave, as the capacity of consumption to raise life expectancy is lower once we are closer to the healthy consumption level.

\footnotetext{
${ }^{15}$ Hence the life expectancy at birth of cohort $t$ is here equal to $1+\pi_{t+1}$.

${ }^{16}$ Naturally, making survival depend on consumption rather than BMI is a shortcut. We could add BMI explicitly in the model, and make it increasing in consumption, without affecting our results. Hence $c^{*}$ would then be the consumption that yields the BMI level at which mortality is the lowest (i.e. the minimum of Waaler's curves).

${ }^{17}$ Note that a richer modelling would add another ingredient: the capacity of the human body to absorb food, and the ability of physiology to adjust over time (see Fogel, 1994).
} 
Production Firms at time $t$ produce an output $Y_{t}$ by means of a capital stock $K_{t}$ and a labour force $L_{t}$, according to a Cobb-Douglas technology: ${ }^{18}$

$$
Y_{t}=A K_{t}^{\alpha} L_{t}^{1-\alpha}
$$

where $0<\alpha<1$ and $A>0$. We assume also a full depreciation of capital after one period of use.

In intensive terms, the production process can be rewritten as

$$
y_{t}=A k_{t}^{\alpha}
$$

where $k_{t}$ is the capital per worker.

Factors are paid at their marginal productivities:

$$
\begin{aligned}
& R_{t}=\alpha A k_{t}^{\alpha-1} \\
& w_{t}=(1-\alpha) A k_{t}^{\alpha}
\end{aligned}
$$

where $R_{t}$ is 1 plus the interest rate, while $w_{t}$ is the wage rate.

The savings decision Each young adult at time $t$ makes a single decision: the amount he saves for his old days (i.e. $s_{t}$ ), and, as a consequence of his budget constraint, his consumption when being young $c_{t}$. For analytical convenience, it is also assumed, as in Chakraborty (2004), that a perfect annuity market exists, which yields an actuarially fair return $\tilde{R}_{t+1} \equiv \frac{R_{t+1}}{\pi_{t+1}}$. That return depends on the actual survival probability to the old age, $\pi_{t+1}$.

In our model, the actual survival probability $\pi_{t+1}$ depends on the consumption when being young $c_{t}$. However, to be in line with the microeconomics of overconsumption, it is assumed here that the agent does not, when he chooses his consumption pattern, internalize the impact of his choice on his survival prospects. On the contrary, the agent takes the future survival prospects as given, and thus independent from his behaviour. More precisely, agents' decisions are here assumed to be based on myopic anticipations about $\pi_{t+1}$ (i.e. $\left.\pi_{t+1}^{e}=\pi_{t}\right)$. This amounts to assuming that agents make their decision on the basis of the currently available period life-table, which is quite realistic. ${ }^{19}$

Thus, under logarithmic temporal utility, and provided the utility of being dead is normalized to zero, the problem of each young adult is to maximize

$$
\log \left(w_{t}-s_{t}\right)+\beta \pi_{t+1}^{e} \log \left(\frac{R_{t+1} s_{t}}{\pi_{t+1}^{e}}\right)
$$

where $\beta$ is a time preference factor $(0 \leq \beta \leq 1)$, while $\pi_{t+1}^{e}$ is the subjective probability of survival to the second period.

Note that, according to equation (6), the agent, when choosing how much to save, uses also the current period life-table to compute the return on annuities, i.e. he takes $\frac{R_{t+1}}{\pi_{t}}$ rather than $\frac{R_{t+1}}{\pi_{t+1}}$ as the return of his savings. That mistake is

\footnotetext{
${ }^{18}$ Note that assuming an $A K$ technology instead would not affect the major properties of the model, as long as the savings decision is based on a life-cycle framework, and is thus affected by the expected lifetime of agents (in that case worker-owners).

${ }^{19}$ At time $t$, the period life expectancy (i.e. the usual life expectancy measure) is $1+\pi_{t}$, whereas the cohort life expectancy of cohort $t$ is $1+\pi_{t+1}$. It is only under constant survival conditions that the two life expectancies are equal.
} 
assumed for the sake of coherency: individuals could hardly perfectly anticipate their life expectancy at one place in their objective function, but not at another. However, it should be stressed that equation (6) does not require the providers of annuities to be myopic: as stated above, the actual annuity return is actuarially fair (i.e. equal to $\frac{R_{t+1}}{\pi_{t+1}}$ ), so that providers of annuities make neither profits nor losses. Equation (6) merely assume that individuals, at the time of their savings decision, do not anticipate the right return on savings.

The first-order condition for optimal savings yields

$$
s_{t}=\frac{\beta \pi_{t}}{1+\beta \pi_{t}} w_{t}
$$

As usual, savings is increasing with the expected lifetime of the agent. Note here that the time preference parameter and the survival probability play here symmetrically, as these both raise the propensity to save.

Finally, it should be noted that, thanks to the logarithmic utility function, the savings decision is not affected at all by the return on savings, so that the fact that the agent takes $\frac{R_{t+1}}{\pi_{t}}$ rather than $\frac{R_{t+1}}{\pi_{t+1}}$ as a return of his savings has no influence on the amounts saved. In other words, if the agent, at the time of his savings decision, could perfectly anticipate the survival probability and the actual return on savings, this would not change anything to his savings decision.

\section{$3 \quad$ Steady-state equilibria}

Let us now study the long-run dynamics of the economy. Given the replacement fertility and the full depreciation of capital, the capital market equilibrium condition is

$$
k_{t+1}=s_{t}
$$

Hence, by substituting for the wage into optimal savings, one has:

$$
k_{t+1}=\frac{\beta \pi_{t}}{1+\beta \pi_{t}} A(1-\alpha) k_{t}^{\alpha}
$$

Obviously, if $k_{t}=0$, we have $k_{t+1}=0$, whatever $\pi_{t}$ is. Hence, in the $\left(\pi_{t}, k_{t}\right)$ space, the $k k$ locus, that is, the combinaisons of $k_{t}$ and $\pi_{t}$ such that $k_{t}$ is constant over time, includes the horizontal axis, i.e., all points $\left(\pi_{t}, 0\right)$.

Imposing $k_{t+1}=k_{t} \neq 0$ gives the other part of the $k k$ locus:

$$
k_{t}=\left[\frac{\beta \pi_{t} A(1-\alpha)}{1+\beta \pi_{t}}\right]^{\frac{1}{1-\alpha}} \equiv G\left(\pi_{t}\right)
$$

We have $G(0)=0, G^{\prime}\left(\pi_{t}\right)>0$ and $G(1)<\infty$. Figure 4 illustrates the $k k$ locus in the $\left(\pi_{t}, k_{t}\right)$ space. $^{20}$ Under $k_{t}>0$, the sustainable level of capital is unique, and is increasing in $\pi_{t}$ : the higher the life expectancy $1+\pi_{t}$ is, the higher the sustainable level of capital is. Levels of $k_{t}$ higher than the $k k$ locus cannot, given the prevailing survival conditions, be reproduced over time. Inversely, levels of capital lower than the $k k$ locus lead to a larger capital at the next period (as a high life expectancy implies here a large propensity to save).

${ }^{20}$ We have $A=25, \alpha=0.30, \beta=0.40, c^{*}=20$ and $\eta=0.01$. 


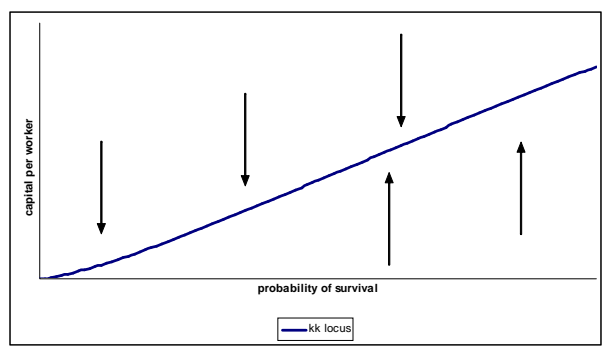

Figure 4: The $k k$ locus

Figure 4 illustrates the relation between the time horizon of agents and the sustainable level of capital. In particular, for bad survival conditions (i.e. a low $\pi_{t}$ ), only extremely low capital levels can be reproduced over time.

Let us now derive the $\pi \pi$ locus, that is, the combinaisons of $k_{t}$ and $\pi_{t}$ such that $\pi_{t}$ is constant over time. From the survival function, we have

$$
\pi_{t+1}=\frac{1}{1+\eta\left(c^{*}-\left(\frac{1}{1+\beta \pi_{t}} A(1-\alpha) k_{t}^{\alpha}\right)\right)^{2}}
$$

Imposing $\pi_{t+1}=\pi_{t}$ gives the $\pi \pi$ locus. Because of the squared bracket at the denominator of the survival function, there exists, in general, not one, but two levels of capital that maintain $\pi_{t}$ at a constant level. These are given by:

$$
\begin{aligned}
& k_{t}=\left[\left(c^{*}-\left(\frac{1-\pi_{t}}{\eta \pi_{t}}\right)^{1 / 2}\right) \frac{1+\beta \pi_{t}}{A(1-\alpha)}\right]^{\frac{1}{\alpha}} \equiv H_{1}\left(\pi_{t}\right) \\
& k_{t}=\left[\left(c^{*}+\left(\frac{1-\pi_{t}}{\eta \pi_{t}}\right)^{1 / 2}\right) \frac{1+\beta \pi_{t}}{A(1-\alpha)}\right]^{\frac{1}{\alpha}} \equiv H_{2}\left(\pi_{t}\right)
\end{aligned}
$$

If $\pi_{t}=1$, then $H_{1}(1)=H_{2}(1)$, as there is a unique level of capital that makes $\pi_{t}$ constant at its maximal level, and that level of $k_{t}$ is such that consumption equals $c^{*}$. Moreover, we have also, for $\bar{\pi} \equiv \frac{1}{1+\eta c^{* 2}} \leq \pi_{t}<1$, that $H_{1}\left(\pi_{t}\right)<$ $H_{2}\left(\pi_{t}\right) .{ }^{21}$ Hence, we shall call $H_{1}\left(\pi_{t}\right)$ the low branch of the $\pi \pi$ locus, and $H_{2}\left(\pi_{t}\right)$ the high branch of the $\pi \pi$ locus. As it is easy to check, those two branches intersect only at $\pi_{t}=1$. Note also that, while we have $\lim _{\pi_{t} \rightarrow 0} H_{2}\left(\pi_{t}\right)=+\infty$ for any value of the parameter $\alpha$, we have that $\lim _{\pi_{t} \rightarrow 0} H_{1}\left(\pi_{t}\right)=+\infty$ only in the special case where $1 / \alpha$ is an even integer number, whereas that limit is not defined otherwise. ${ }^{22}$

Regarding the shape of $H_{1}\left(\pi_{t}\right)$, we have, in the general case where $1 / \alpha$ is not an even integer, that, if $(1 / \alpha)-1$ is not an even integer, that $H_{1}^{\prime}\left(\pi_{t}\right)<0$ for $\pi_{t}<\bar{\pi}$ and $H_{1}^{\prime}\left(\pi_{t}\right)>0$ for $\pi_{t}>\bar{\pi}$. At $\pi_{t}=\bar{\pi}$, we have $H_{1}^{\prime}\left(\pi_{t}\right)=0$.

\footnotetext{
${ }^{21}$ The same claim cannot be made for $\pi_{t}<\bar{\pi}$, because $H_{1}\left(\pi_{t}\right)$ is not defined in that case for most values of the parameter $\alpha$ (see below).

${ }^{22}$ Actually, $H_{1}\left(\pi_{t}\right)$ is not well defined for $\pi_{t}<\bar{\pi}$, since the factor in brackets in $H_{1}\left(\pi_{t}\right)$ is negative for $\pi_{t}<\bar{\pi}$, so that the whole expression becomes undefined except when $1 / \alpha$ is an even integer number.
} 
However, if $(1 / \alpha)-1$ is an even integer (i.e. if $1 / \alpha$ is an odd integer), we have $H_{1}^{\prime}\left(\pi_{t}\right)>0$ for $\pi_{t} \in[\bar{\pi}, 1]$. In the special case where $1 / \alpha$ is an even integer, we have $H_{1}^{\prime}\left(\pi_{t}\right)<0$ for $\pi_{t}<\bar{\pi}, H_{1}^{\prime}\left(\pi_{t}\right)=0$ for $\pi_{t}=\bar{\pi}$ and $H_{1}^{\prime}\left(\pi_{t}\right)>0$ for $\pi_{t}>\bar{\pi}$.

As far as $H_{2}\left(\pi_{t}\right)$ is concerned, we have $H_{2}^{\prime}\left(\pi_{t}\right)<0$ for any $\pi_{t}$ such that

$$
\left(\frac{1}{\eta}\right)^{\frac{1}{2}}\left(\frac{1-\pi_{t}}{\pi_{t}}\right)^{\frac{1}{2}}\left[\frac{1}{2} \frac{\left(1+\beta \pi_{t}\right)}{\pi_{t}\left(1-\pi_{t}\right)}-\beta\right]>c^{*}
$$

If $\pi_{t}<\bar{\pi}$, we have $\left(\frac{1}{\eta}\right)^{\frac{1}{2}}\left(\frac{1-\pi_{t}}{\pi_{t}}\right)^{\frac{1}{2}}>c^{*}$. Hence, given that the term in brackets lies in the interval $[2,+\infty]$, it must be the case that $H_{2}^{\prime}\left(\pi_{t}\right)<0$. Alternatively, if $\pi_{t}>\bar{\pi}$, we have $\left(\frac{1}{\eta}\right)^{\frac{1}{2}}\left(\frac{1-\pi_{t}}{\pi_{t}}\right)^{\frac{1}{2}}<c^{*}$, and so it may be the case that $H_{2}^{\prime}\left(\pi_{t}\right)>0$.

Figures $5 \mathrm{a}$ and $5 \mathrm{~b}$ illustrate the form of the $\pi \pi$ locus in the $\left(\pi_{t}, k_{t}\right)$ space, for the general case (i.e. $1 / \alpha$ is not an even integer) and the special case (i.e. $1 / \alpha$ is an even integer). ${ }^{23}$ For $\pi_{t}<1$, there exists an interval of levels for capital per worker that allow a growth of life expectancy over time. That interval lies between the two branches of the $\pi \pi$ locus. Levels of $k_{t}$ outside that interval lead to a fall of life expectancy. Such a fall can arise either because $k_{t}$ is too low (bottom of the phase diagram), or because $k_{t}$ is too high (top of the phase diagram). In the former case, consumption is lower than the level that would maintain life expectancy unchanged. As a consequence, $\pi_{t}$ must fall, as only a lower survival probability can be sustained for such a low consumption level. In the latter case, a fall of $\pi_{t}$ arises because $k_{t}$ is too high: consumption exceeds the level that would maintain $\pi_{t}$ constant, explaining the fall of $\pi_{t}$.

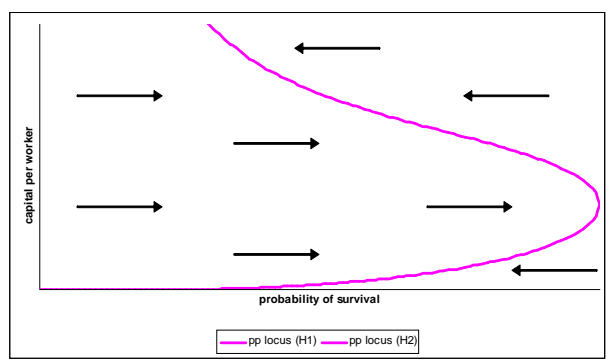

Fig. 5a: The $\pi \pi$ locus (general case)

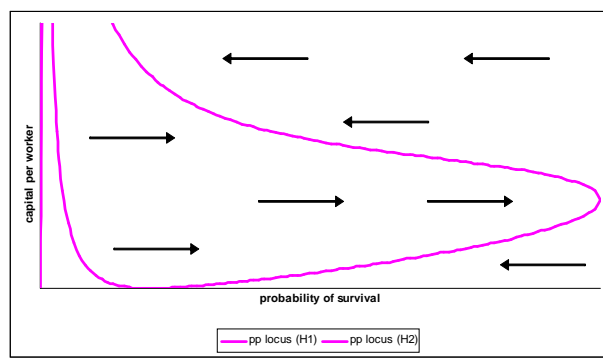

Fig. 5b: The $\pi \pi$ locus (special case)

There can be three distinct kinds of stationary equilibria in the economy under study, as the intersections of the two loci can occur either on the low branch of the $\pi \pi$ locus, or on the high branch of the $\pi \pi$ locus, or at the intersection of the two branches, i.e. at $\pi_{t}=1$. In the rest of this paper, we will coin the different types of equilibria as follows. The first type of intersection will be called an underconsumption equilibrium (UC), as consumption at such an equilibrium is below the healthy consumption level $c^{*}$. The second type of equilibrium will be referred to as an overconsumption equilibrium (OC), as consumption exceeds

\footnotetext{
${ }^{23}$ On Figure 5a, we have $A=25, \alpha=0.30, \beta=0.40, c^{*}=20$ and $\eta=0.01$. Same values on Figure 5b, except $\alpha=0.50$.
} 
$c^{*}$ at that equilibrium. The third type of intersection will be called a healthy consumption equilibrium ( $\mathrm{HC})$, as we have $c_{t}=c^{*}$ at that equilibrium.

Let us now use the properties of the $k k$ locus and the $\pi \pi$ locus to study the existence of steady-state equilibria. Proposition 1 summarizes our results. ${ }^{24}$

Proposition 1 Denote $\left[\frac{\beta A(1-\alpha)}{1+\beta}\right]^{\frac{1}{1-\alpha}}$ by $k^{*}$ and $\left[c^{*} \frac{1+\beta}{A(1-\alpha)}\right]^{\frac{1}{\alpha}}$ by $\Omega .^{25}$

- General case: $1 / \alpha$ is not an even integer:

(1) If $k^{*}<\Omega$, there exist at least two UC equilibria: $(\bar{\pi}, 0)$ and $\left(\pi_{1}, k_{1}\right)$, with $0<\bar{\pi}<\pi_{1}<1$ and $0<k_{1}<k^{*}$.

(2) If $k^{*}>\Omega$, there exist at least one UC equilibrium $(\bar{\pi}, 0)$ and one $O C$ equilibrium $\left(\pi_{2}, k_{2}\right)$, where $0<\bar{\pi} \lessgtr \pi_{2}<1$ and $0<k^{*}<k_{2}$.

(3) If $k^{*}=\Omega$, there exist at least one UC equilibrium $(\bar{\pi}, 0)$ and exactly one $H C$ equilibrium $\left(1, k^{*}\right)$.

- Special case: $1 / \alpha$ is an even integer:

(4) If $k^{*}<\Omega$, there exists at least three UC equilibria $(\bar{\pi}, 0),\left(\pi_{3}, k_{3}\right)$ and $\left(\pi_{4}, k_{4}\right)$, with $0<\pi_{3}<\bar{\pi}<\pi_{4}<1$ and $0<k_{3}<k_{4}<k^{*}$.

(5) If $k^{*}>\Omega$, there exists at least two UC equilibria $(\bar{\pi}, 0)$ and $\left(\pi_{5}, k_{5}\right)$, and at least one $O C$ equilibrium $\left(\pi_{6}, k_{6}\right)$, with $0<\pi_{5}<\bar{\pi}, \bar{\pi} \lessgtr \pi_{6}<1$ and $0<k_{5}<k^{*}<k_{6}$.

(6) If $k^{*}=\Omega$, there exists at least two UC equilibria $(\bar{\pi}, 0)$ and $\left(\pi_{7}, k_{7}\right)$, and exactly one $H C$ equilibrium $\left(1, k^{*}\right)$, with $0<\pi_{7}<\bar{\pi}<1$ and $0<k_{7}<k^{*}$.

Proof. See the Appendix.

Proposition 1 states that the minimal number of steady-state equilibria depends on the structural parameters of the economy. ${ }^{26}$ In cases (4) to (6), where $1 / \alpha$ is an even integer, there must exist a stationary equilibrium in the left corner of the $\left(\pi_{t}, k_{t}\right)$ space, because the low branch of the $\pi \pi$ locus is decreasing for levels of $\pi_{t}$ inferior to $\bar{\pi}$, unlike what prevails under cases (1) to (3), where $1 / \alpha$ is not an even integer. This is the reason why cases (4) to (6) admit a higher minimal number of steady-state equilibria than cases (1) to (3).

Regarding the distinction between, on the one hand, cases (1) and (4), and, on the other hand, cases (2) and (5), this lies in the types of steady-state equilibria that exist in each case. The stationary equilibria $(\bar{\pi}, 0),\left(\pi_{1}, k_{1}\right),\left(\pi_{3}, k_{3}\right)$, $\left(\pi_{4}, k_{4}\right)$ and $\left(\pi_{5}, k_{5}\right)$ are underconsumption equilibria, i.e. equilibria located on the low branch of the $\pi \pi$ locus. On the contrary, $\left(\pi_{2}, k_{2}\right)$ and $\left(\pi_{6}, k_{6}\right)$ are overconsumption equilibria, i.e. equilibria located on the high branch of the $\pi \pi$ locus. While this constitutes a significant difference, this does not allow us, however, to deduce whether life expectancy is higher at an underconsumption equilibrium or at an overconsumption equilibrium, as the level of life expectancy depends on the distance between consumption and healthy consumption.

Overconsumption equilibria $\left(\pi_{2}, k_{2}\right)$ and $\left(\pi_{6}, k_{6}\right)$ are, ceteris paribus, more likely to prevail in economies with a higher productivity (i.e. with a high $A$ ). Similarly, it is clear, from Proposition 1, that overconsumption equilibria are more plausible in economies where the time preference factor $\beta$ is close to unity.

\footnotetext{
${ }^{24}$ For the ease of presentation, "equilibrium" refers here to "steady-state equilibrium".

${ }^{25} k^{*}$ is the level of $k$ that coincides with a consumption equal to the "healthy" level $c^{*}$

${ }^{26}$ Proposition 1 concerns only the minimal number of equilibria because the existence proof relies on the limits of $G\left(\pi_{t}\right), H_{1}\left(\pi_{t}\right)$ and $H_{2}\left(\pi_{t}\right)$ for $\pi_{t}$ tending towards $0, \bar{\pi}$ and 1 . Additional assumptions on the second-order derivatives of the loci are needed to be able to make statements about the actual number of intersections of the two loci (see the Appendix).
} 
Under a low impatience, we have $G(1)>H_{1}(1)=H_{2}(1)$, which leads to an overconsumption equilibrium. Finally, economies where the healthy consumption level $c^{*}$ is, because of external reasons, lower are also more likely to exhibit an overconsumption equilibrium.

Cases (1), (2), (4) and (5) of Proposition 1 are illustrated on Figures 6a to $6 \mathrm{~d}$, which show the $k k$ locus and the $\pi \pi$ locus in the $\left(\pi_{t}, k_{t}\right)$ space..$^{27}$ In order to give us some clues regarding the local stability of stationary equilibria, those figures exhibit dynamic arrows, showing the direction of change over time. In each case, there exists a vast area of the $\left(\pi_{t}, k_{t}\right)$ space where both capital per worker and life expectancy are growing. Note, however, that the size of that area varies strongly across the cases. More importantly, the extent to which an economy with initial conditions $\left(\pi_{0}, k_{0}\right)$ can end up in that area varies across cases. Under cases (4) and (5), there exists a large area, in the bottom left corner of the phase diagram, where both capital per worker and life expectancy are falling. In those cases, the intermediate equilibrium is unstable, and acts like a threshold, below which economies are condamned to stagnate, with low output, consumption and life expectancy.

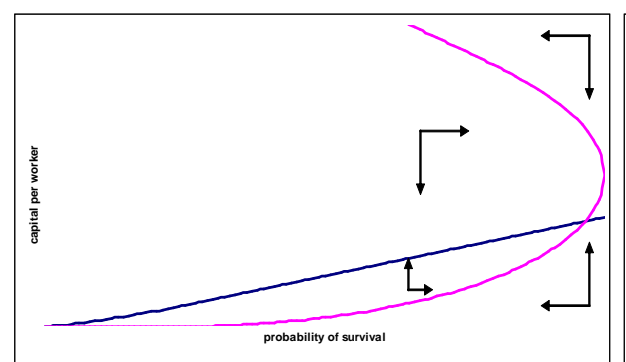

Figure 6a: Case (1)

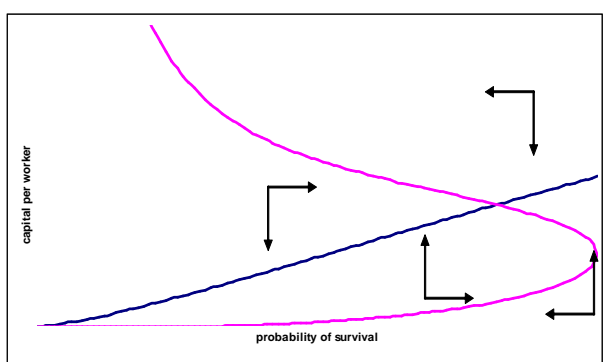

Figure 6b: Case (2)

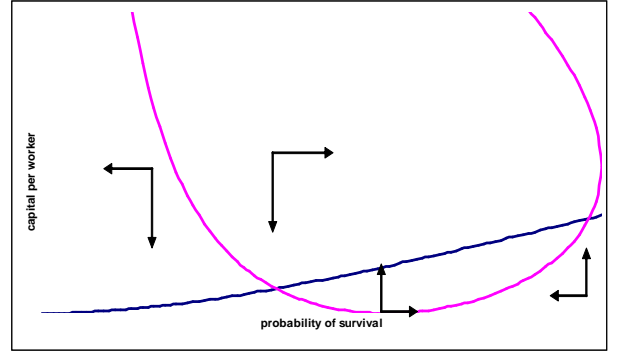

Figure 6c: Case (4)

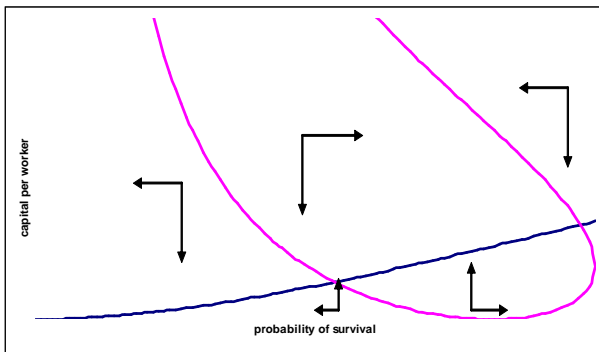

Figure 6d: Case (5)

\footnotetext{
${ }^{27}$ On Figure $6 \mathrm{a}$, we have $A=20, \alpha=0.30, \beta=0.40, c^{*}=20$, and $\eta=0.01$. On Figure $6 \mathrm{~b}$, we have $A=25, \alpha=0.30, \beta=0.40, c^{*}=20$, and $\eta=0.01$. On Figure $6 \mathrm{c}$, we have $A=25$, $\alpha=0.50, \beta=0.40, c^{*}=20$, and $\eta=0.001$. On Figure 6d, we have $A=20, \alpha=0.50$, $\beta=0.40, c^{*}=15$, and $\eta=0.001$.
} 
Whereas this discussion gives us some clues about the instability of some equilibria, it should be reminded, however, that a mere look at a phasis diagram does not suffice to provide accurate results on the stability of equilibria. For instance, on Figure 6b, it is not possible to see, on the mere basis of graphical analysis, whether the equilibrium is stable or not: even though the dynamic arrows seem to point to the equilibrium, nothing insures the convergence to that equilibrium. A formal analysis of stability is required. Such an analysis is carried out in the Appendix. Proposition 2 summarizes our results. ${ }^{28}$

Proposition 2 Suppose the economy admits a steady-state equilibrium $(\pi, k)$. The conditions

$$
\begin{gathered}
1+\eta\left(c^{*}-c\right)^{2}>2 \eta\left(c^{*}-c\right) c \frac{\beta+\alpha\left[1+\beta+\eta\left(c^{*}-c\right)^{2}\right]}{(1+\alpha)\left(1+\eta\left(c^{*}-c\right)^{2}+\beta\right)} \\
1+\eta\left(c^{*}-c\right)^{2}>-2 \eta\left(c^{*}-c\right) c \frac{\beta-\alpha\left[1+\beta+\eta\left(c^{*}-c\right)^{2}\right]}{(1-\alpha)\left(1+\eta\left(c^{*}-c\right)^{2}+\beta\right)} \\
1+\eta\left(c^{*}-c\right)^{2}>-2 \eta\left(c^{*}-c\right) c \alpha
\end{gathered}
$$

are necessary and sufficient for the local stability of that equilibrium.

Proof. See the Appendix.

Those conditions concern the local stability only, and not the global stability, because, as this was shown above, there exist, in each of the cases (1) to (6), at least two steady-state equilibria, so that no condition can guarantee a convergence towards a particular equilibrium from any initial situation $\left(\pi_{0}, k_{0}\right)$.

The stability conditions of Proposition 2 are general (i.e. valid for all cases of Proposition 1), and, as such, are not simple to interpret. ${ }^{29}$ The difficulty to interpret those conditions is reinforced by the fact that those conditions depend on the equilibrium levels of consumption. Despite those difficulties, the stability conditions of Proposition 2 can inform us on the central role played by the parameter $\eta$, which captures the sensitivity of life expectancy to the consumption behaviour. Clearly, if $\eta$ tends to 0 , the stability conditions become respectively $1>0,1>0$ and $1>0$, which are all true. However, larger values of $\eta$ make the local stability of the stationary equilibrium less likely, since these imply large changes in life expectancy as a reaction to small changes in consumption. How reactive life expectancy is to consumption changes is thus a major determinant of (in)stability. Moreover, the conditions of Proposition 2 reveal also that a major determinant of stability is the distance of the equilibrium consumption from the healthy consumption $c^{*}$. When that distance tends towards 0 , the three conditions tend to $1>0,1>0$ and $1>0$, which are trivially satisfied.

In order to have a more concrete idea of the conditions under which stability prevails, let us now focus on the case of an overconsumption equilibrium. If one

\footnotetext{
${ }^{28}$ Those conditions are stated in terms of the equilibrium level of $c$. The reason why this is so has to do with the absence of closed-form analytical solutions for the equilibrium levels of $k$ and $\pi$, which prevents us from deriving conditions including parameters only (see below).

${ }^{29}$ One exception concerns the stability of the steady-state equilibrium $(\bar{\pi}, 0)$. In that case $c=0$ and it is straightforward to see that the three conditions of Proposition 2 are satisfied, so that local stability prevails.
} 
substitutes for equilibrium consumption in the stability conditions of Proposition 2 , it can be shown that, for overconsumption equilibria, such as $\left(\pi_{2}, k_{2}\right)$ and $\left(\pi_{6}, k_{6}\right)$, a simple condition guarantees the local stability of the equilibrium.

Proposition 3 Denote $[A(1-\alpha)]^{\frac{1}{1-\alpha}}$ by $\chi$. The condition

$$
\alpha-\frac{2 \eta\left(\frac{1}{1+\beta \pi}\right)^{\frac{2-\alpha}{1-\alpha}} \pi^{\frac{\alpha}{1-\alpha}} \beta^{\frac{1-2 \alpha}{1-\alpha}} \chi\left(c^{*}-\left(\frac{1}{1+\beta \pi}\right)^{\frac{1}{1-\alpha}} \pi^{\frac{\alpha}{1-\alpha}} \beta^{\frac{\alpha}{1-\alpha}} \chi\right)}{\left[1+\eta\left(c^{*}-\left(\frac{1}{1+\beta \pi}\right)^{\frac{1}{1-\alpha}} \pi^{\frac{\alpha}{1-\alpha}} \beta^{\frac{\alpha}{1-\alpha}} \chi\right)^{2}\right]^{2}}<1
$$

is sufficient for the local stability of equilibria $\left(\pi_{2}, k_{2}\right)$ and $\left(\pi_{6}, k_{6}\right)$.

Proof. See the Appendix.

The closer the equilibrium consumption is to the healthy consumption $c^{*}$, the lower the second term of the condition is, making local stability more plausible. ${ }^{30}$ Here again, larger values of $\eta$ make the local stability of the equilibrium less likely. Indeed, if $\eta$ tends towards 0 , the condition collapses to $\alpha<1$, which is trivially satisfied. On the contrary, a larger $\eta$ allows larger fluctuations of life expectancy as a reaction to small consumption changes, which goes against stability. Finally, the time preference parameter $\beta$ is also present in the stability condition. The more impatient agents are (i.e. a lower $\beta$ ), the lower the second term of the condition is, making stability more plausible. ${ }^{31}$ The intuition behind this lies in the mere fact that, if $\beta$ is low, the agent's reactions to a change in their expected time horizon are necessarily of smaller sizes, which favours the stability of the long-run equilibrium. Inversely, a large patience implies high savings changes in reaction to a rise in life expectancy.

Let us conclude this stability analysis by considering the possibility of longrun cycles in the $\left(\pi_{t}, k_{t}\right)$ space. As stated in Proposition 4 , it is only in the presence of an overconsumption equilibrium, that is, in cases (2) and (5) of Proposition 1 , that long-run cycles can arise in the $\left(\pi_{t}, k_{t}\right)$ space. Such cycles are both economic cycles (i.e. in terms of capital, output and consumption) and demographic cycles (i.e. in terms of life expectancy and population size). The existence of long-run cycles is subject to some specific conditions on the structural parameters of the economy.

Proposition 4 There exists no long-run cycle around steady-state equilibria $(\bar{\pi}, 0),\left(\pi_{1}, k_{1}\right),\left(\pi_{3}, k_{3}\right),\left(\pi_{4}, k_{4}\right),\left(\pi_{5}, k_{5}\right),\left(\pi_{7}, k_{7}\right)$ and $\left(1, k^{*}\right)$.

There exist long-run cycles around the steady-states $\left(\pi_{2}, k_{2}\right)$ and $\left(\pi_{6}, k_{6}\right)$ if and only if the following conditions are satisfied:

(i) $\left[\frac{\alpha\left[1+\eta\left(c^{*}-c\right)^{2}\right]-\frac{2 \eta\left(c^{*}-c\right) \beta c}{1+\beta+\eta\left(c^{*}-c\right)^{2}}}{1+\eta\left(c^{*}-c\right)^{2}}\right]^{2}+8 \frac{\alpha \eta\left(c^{*}-c\right) c}{1+\eta\left(c^{*}-c\right)^{2}}<0$

(ii) $\sqrt[2]{\frac{-2 \alpha \eta\left(c^{*}-c\right) c}{1+\eta\left(c^{*}-c\right)^{2}}}=1$

where $c$ takes its equilibrium value.

${ }^{30}$ This can be seen easily when rewriting the above condition as $\alpha-\frac{2 \eta\left(c^{*}-c\right)\left(c \frac{\beta}{1+\beta \pi}\right)}{\left[1+\eta\left(c^{*}-c\right)^{2}\right]^{2}}<1$, where $c$ is the equilibrium consumption level.

${ }^{31}$ Actually, when $\beta$ tends to 0 , the above condition becomes $\alpha<1$ (if $\alpha \leq 1 / 2$ ) and $-\infty<1$ (if $\alpha>1 / 2$ ), which are always satisfied. 
Proof. See the Appendix.

It is easy to see why cycles cannot arise around underconsumption equilibria like $(\bar{\pi}, 0),\left(\pi_{1}, k_{1}\right),\left(\pi_{3}, k_{3}\right),\left(\pi_{4}, k_{4}\right)$ or $\left(\pi_{5}, k_{5}\right)$. Indeed, in those cases, the condition (i) is necessarily violated, as $c<c^{*}$. It is thus only at overconsumption equilibria, like $\left(\pi_{2}, k_{2}\right)$ and $\left(\pi_{6}, k_{6}\right)$, that condition (i) can be satisfied, and if condition (ii) is also true, then a cycle exists around the steady-state.

Note, here again, the crucial role played by the parameter $\eta$. If $\eta$ is close to zero, cycles cannot occur, as conditions (i) and (ii) are necessarily violated. Moreover, for too high levels of $\eta$, it is condition (ii) that would not be satisfied: the economy would diverge in the long-run, without exhibiting a cycle. Thus, the existence of long-run cycles requires a particular set of conditions, including a sensitivity of life expectancy to consumption behaviour that is neither too small, neither too large.

While Proposition 4 informs us about the general conditions under which long-run cycles exist in the $\left(\pi_{t}, k_{t}\right)$ space, it is difficult to know a priori whether conditions (i) and (ii) are strong or weak, and whether these are compatible with standard values for the parameters of the economy. One reason why the conditions of Proposition 4 are hard to interpret has to do with the fact that these are written in terms of the equilibrium consumption level, whereas, ideally, those conditions should include structural parameters only. However, as stated above, there is no closed-form solution for equilibrium level of $c$ (i.e. in terms of parameters only), and this is why we opted here for that presentation. Obviously, this analytical discussion needs to be complemented by numerical simulations, to be able to see to what extent the conditions mentionned in Proposition 4 can be satisfied under plausible values of the structural parameters of the economy. The task of the next section is to discuss this by means of numerical simulations.

\section{$4 \quad$ Numerical illustrations}

Given that the model studied in Sections 2 and 3 does not admit a closed-form analytical solution for equilibrium levels of consumption $c$ and life expectancy $1+\pi$, the interpretation of the conditions under which stability or instability and possibly cycles - prevail is difficult. Hence, it makes sense to complement the above analytical developments by a numerical section.

The goal of this section is to illustrate numerically the dynamics of production and longevity in our economy, and to show in which cases long-run cycles in output and longevity occur. For those purposes, we will focus here on the cases of advanced economies, i.e. on economies with a high productivity. Hence, the equilibria under study belong here to the cases (2) and (5) of Proposition 1 (i.e. steady-states $\left(\pi_{2}, k_{2}\right)$ and $\left.\left(\pi_{6}, k_{6}\right)\right)$. We will rely on the following benchmark values for the structural parameters of the economy. ${ }^{32}$

\footnotetext{
${ }^{32}$ Note that the time preference parameter $\beta$, which is fixed to 0.40 , is slightly larger than the usual value of 0.30 . Actually, 0.30 is generally used, as this coincides with a quarterly discount factor of 0.99 . Here we rely on a higher value for $\beta$, as there is already some "natural" discounting through the survival probability.
} 


\begin{tabular}{cc}
\hline \hline Parameters & values \\
\hline \hline & \\
$A$ & 30 \\
$\alpha$ & 0.300 \\
$\beta$ & 0.400 \\
$\eta$ & 0.010 \\
$c^{*}$ & 30 \\
\hline \hline
\end{tabular}

We shall also take, as initial conditions, $k_{0}=0.1$ and $\pi_{0}=0.05$ (for periods of 30 years, this coincides with an initial life expectancy of about 61.5 years). It is easy to check that, under those parameters values, we have $\left[c^{*} \frac{1+\beta}{A(1-\alpha)}\right]^{\frac{1}{\alpha}}<$ $\left[\frac{\beta A(1-\alpha)}{1+\beta}\right]^{\frac{1}{1-\alpha}}$, so that we are in case $(2)$.

In the light of the discussions in Section 3, one can expect that the dynamics of production and longevity depends on the parameter $\eta$, which determines the reaction of longevity when consumption departs from its healthy level. That intuition is confirmed by Figures 7a-7d, which show the dynamics of the economy in the $\left(\pi_{t}, k_{t}\right)$ space. Under low values of $\eta$, the convergence to the long-run equilibrium is monotone, except when the economy is very close to the long-run equilibrium (see Figures $7 \mathrm{a}$ and $7 \mathrm{~b}$ ). A converging spiral holds under $\eta=0.020$ (Figure 7c). However, under $\eta=0.030$ (Figure 7d), there is a non-converging cycle, as the economy satisfies the conditions of Proposition 4.

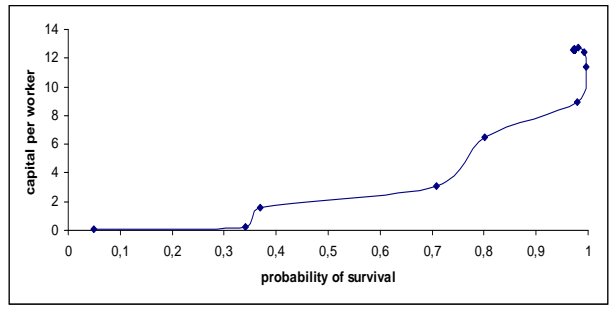

Figure $7 \mathrm{a}: \eta=0.005$

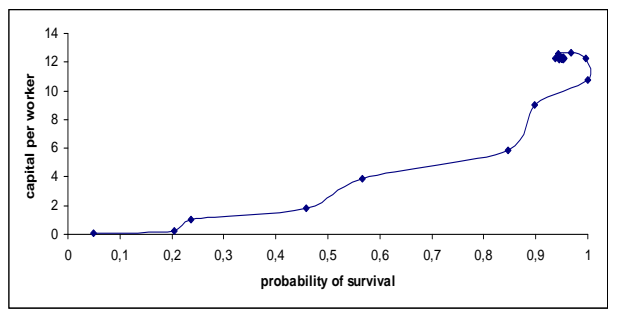

Figure $7 \mathrm{~b}: \eta=0.010$

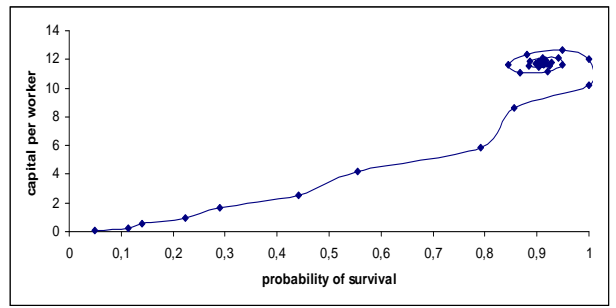

Figure 7c: $\eta=0.020$

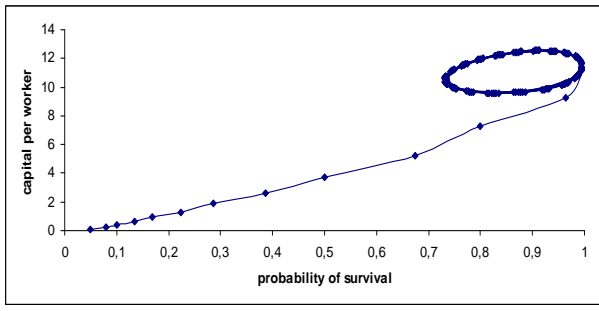

Figure $7 \mathrm{~d}: \eta=0.030$

An interesting feature of Figures $7 \mathrm{c}$ and $7 \mathrm{~d}$ is that, if we look at the first 10 periods of time, the dynamics is quasi identical whatever $\eta$ equals 0.020 or 0.030. Given the substantial length of each period (equal to about 30 years), 
it follows from this that empirical evidence covering about three centuries of data on output and longevity would not help us to distinguish between $\eta=$ 0.020 and $\eta=0.030$, even though the long-run dynamics induced by those two parametrizations are very different. This constitutes a quite negative result, as one can thus hardly, on the basis of existing data sets, discriminate between different levels of $\eta$, and, hence, detect the possible existence of long-run cycles.

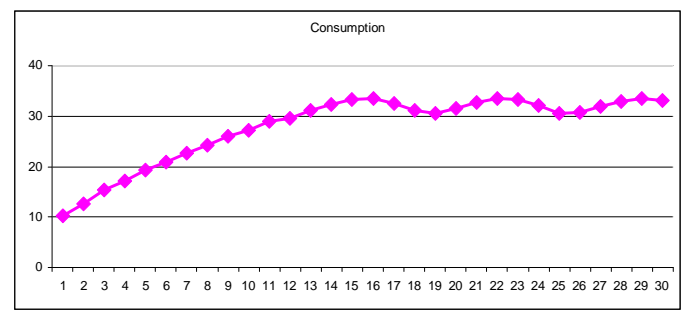

Figure 8a: the dynamics of consumption

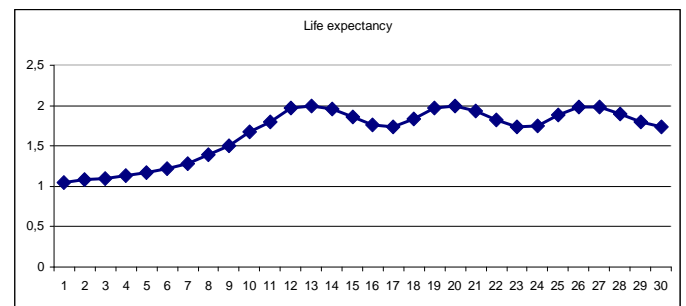

Figure 8b: the dynamics of life expectancy

In order to understand the mechanism behind long-run cycles, Figures 8a and $8 \mathrm{~b}$ show the time series of consumption and life expectancy under $\eta$ equal to 0.030. As long as consumption is below the healthy consumption level $c^{*}$ (equal to 30 ), the capital accumulation process makes consumption grow towards $c^{*}$, implying a growth of life expectancy. Thanks to the horizon effect, a high life expectancy keeps on reinforcing capital accumulation, which raises consumption beyond $c^{*}$. This overconsumption leads to a fall of life expectancy. For a low level of $\eta$, departures from $c^{*}$ cause only small changes in life expectancy, which will have minor effects on capital accumulation, and, thus, on future consumption and life expectancy. Hence, for low levels of $\eta$, there will be a convergence of $c_{t}$ and $\pi_{t}$ towards the long-run equilibrium. However, for larger values of $\eta$, the fall of life expectancy induced by an excessive consumption is significant. Due to the horizon effect, that fall of life expectancy reduces capital accumulation, and, in fine, consumption, which falls down towards its healthy level. This raises life expectancy again, which increases savings and capital accumulation, and so forth. A large $\eta$, by implying strong upwards and downwards reactions of life expectancy to consumption and vice versa, is thus the cause of the instability, which takes here the form of a cycle. ${ }^{33}$

\footnotetext{
${ }^{33}$ Note that larger levels of $\eta$ can lead to diverging spirals around the equilibrium.
} 
Let us now examine the robustness of those results to the calibration of the other parameters. As shown on Figures 9a-9b, a slight change in the level of healthy consumption $c^{*}$ has large effects on the long-run dynamics of longevity and capital. On Figure 9a, we can see that, under $\eta=0.030$, cycles disappear once $c^{*}$ is raised from 30 to 31 . On the contrary, Figure $9 \mathrm{~b}$ shows that cycles become larger once healthy consumption is slightly reduced.

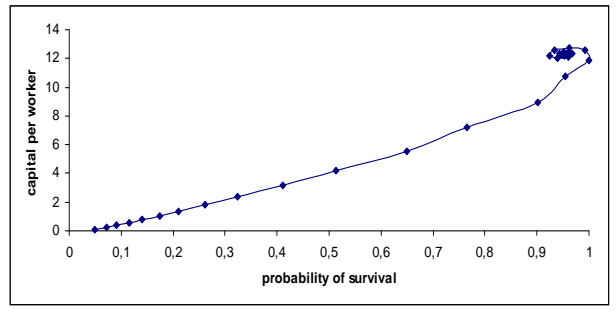

Figure 9a: $\eta=0.030, c^{*}=31$

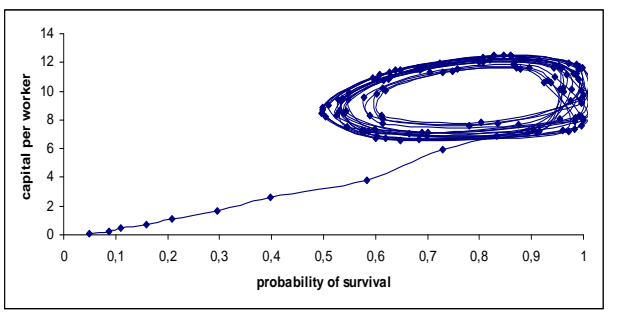

Figure 9b: $\eta=0.030, c^{*}=29$

Beyond $\eta$ and $c^{*}$, other parameters enter the stability condition of Proposition 3 , and can be expected to influence the overall dynamics of the economy. Take, for instance, the time preference parameter $\beta$. The higher it is, the stronger the horizon effect is, and so the stronger the effect of survival conditions on capital accumulation is. Note, however, that numerical simulations under the benchmark values of the other parameters do not point to a significant sensitivity of the long-run dynamics to the level of $\beta .{ }^{34}$

On the contrary, the dynamics of output and longevity is strongly sensitive to the parameter $\alpha$, i.e. the elasticity of output with respect to capital. This influence is obvious in the light of the stability condition of Proposition 3: the higher $\alpha$ is, the less plausible local stability is. As shown on Figure 10a, once $\alpha$ is raised to 0.4 , a level of $\eta$ as low as 0.008 suffices to bring a non-converging cycle, whereas, under $\alpha=0.5$, non-converging cycles appear for $\eta=0.004$ (Figure 10b). Hence, more capital-intensive economies are also likely to exhibit an unstable stationary equilibrium.

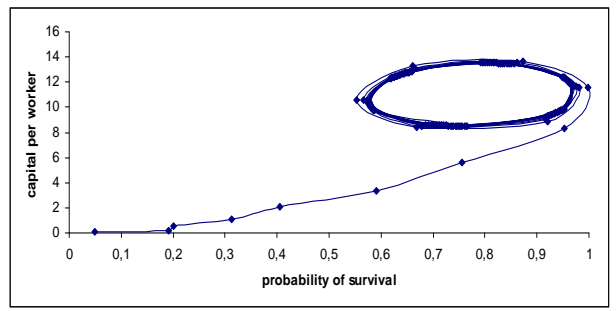

Figure 10a: $\alpha=0.4, \eta=0.008$

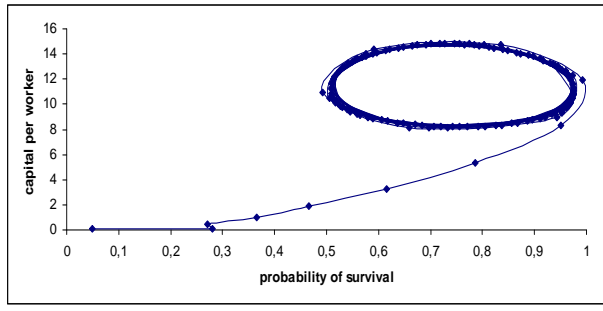

Figure 10b: $\alpha=0.5, \eta=0.004$

\footnotetext{
${ }^{34}$ But this does not mean that this parameter is benign, as it influences naturally the position of the steady-state equilibrium levels of output and life expectancy. Because of space constraints, those simulations are not included here.
} 
Finally, note that, although the productivity parameter $A$ does not explicitly enter the stability condition in Proposition 2, it tends, however, to have a significant influence on the dynamics of output and longevity. As shown on Figure $7 \mathrm{~b}$, there is, under $\eta$ equal to its benchmark value of 0.01 , a non-monotone convergence of the economy to the steady-state equilibrium under $A=30$. Figures $11 \mathrm{a}$ and $11 \mathrm{~b}$ show that changes in $A$ affect the dynamics significantly: the convergence becomes monotone in $\pi_{t}$ and $k_{t}$ under $A=25$ (Figure 11a), and there exists a non-converging cycle under $A=35$ (Figure 11b).

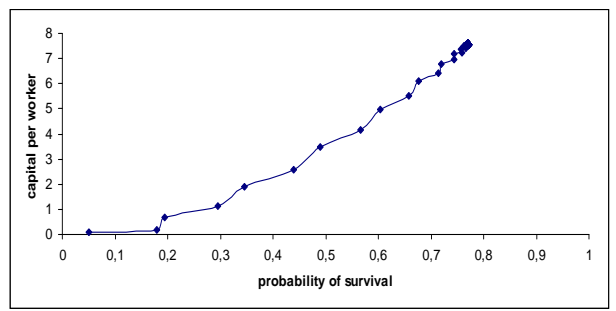

Figure 11a: $A=25$

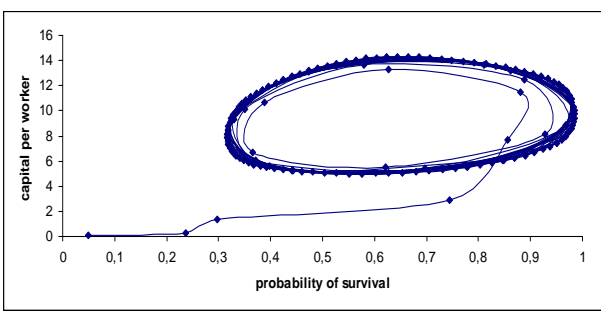

Figure 11b: $A=35$

Hence, more productive economies are more likely to exhibit a cyclical dynamics than less productive economies. Indeed, in more productive economies, the impact of capital accumulation in terms of output and consumption is larger. As a consequence, the reactions of longevity to capital accumulation are also larger, reinforcing the likelihood of cycles.

That result is worth being stressed, especially if one thinks that a major limitation of the model developed in Section 2 lies in the absence of technological progress. Figure 11b suggests that if some exogenous technological progress was assumed instead (i.e. a variable $A_{t} \equiv A(1+g)^{t}$ in the production function), the high levels of $A_{t}$ reached after some periods of time would lead the economy to fluctuations in output and life expectancy. Hence the introduction of some exogenous technological progress would reinforce the likelihood of long-run cycles. However, endogenous technological progress may or may not do so, depending on the precise modelling of the determinants of technological change.

To conclude, it should be stressed that those numerical simulations are purely illustrative of the model, but can hardly serve as a test of the validity of the model's assumptions (or of the values of its parameters). The reason why this section cannot serve as a test is twofold. Firstly, actual levels of output and life expectancy have been evolving under the influence of production and medical technologies, which have been shifting over time, contrary to our fixedtechnology model. Hence one could hardly compare the actual time series of output and longevity with the series simulated in this section. Secondly, our numerical simulations suggest that longevity cycles, if these exist, may exhibit long periods. Thus existing data sets on output and life expectancy are likely to be too short to allow a decisive empirical testing. ${ }^{35}$

\footnotetext{
${ }^{35}$ For instance, if the data set shows a monotone evolution of life expectancy over the last three centuries, it is possible to calibrate the present model to get a cycle whose ascending part lasts at least three centuries.
} 


\section{Discussions}

Having shown numerically that long-term cycles can arise in our economy under plausible values of structural parameters, it is worth making here a few observations concerning the origin of those cycles. This will also be the opportunity to connect our study with the literature on economic and demographic cycles.

A first way to interpret the occurence of economic cycles in the present framework consists of noticing that the introduction of endogenous longevity raises the dimension of the dynamic system from 1 to 2 . As this is well known in the literature, such a rise in the dimension of a dynamic system is far from neutral as far as the study of stability is concerned. Actually, even if one takes a simple 2-period OLG model with physical capital, the mere introduction of, let us say, debt (see Farmer, 1986) or family social capital (see de la Croix and Michel, 2002) may suffice to make cycles appear. Hence, in the light of this, it should not come as a surprise that the endogenization of longevity, by raising the dimension of the dynamic system, may lead to long-run cycles.

A second standard ingredient in the existence of cycles is the presence of non-linearities. True, cycles can arise even in simple one-dimensional linear dynamic systems such as Samuelson's (1939) accelerator model. However, whereas cycles appear only under a quite limited set of values for structural parameters in linear models, these are much more likely in models including non-linearities. For instance, Day (1982) examined how cycles can occur in a discrete-time Solow (1956) economy where productivity is reduced by the pollution induced by capital accumulation. The physical capital accumulation process in Day's model can, for particular values of parameters, be represented by means of a logistic equation, which can lead to complex dynamics (including cycles) under particular conditions. That second ingredient of cycles is also present in our model, where the probability of survival to the old age is a non-monotone function of consumption, and takes the form of an inverted logistic function.

Finally, beyond the dimension issue and non-linearities, another way to interpret the occurence of cycles in the present model is related to the existence of myopia. Actually, myopic anticipations have been shown to be a source of long-run fluctuations and cycles even in simple one-good models. For instance, de la Croix and Michel (2002) show, in a two-period OLG model with CobbDouglas production and CIES preferences, that cycles may arise when agents form myopic anticipations regarding the return on savings, unlike what happens under perfect foresight where cycles never occur under those assumptions. ${ }^{36}$ In the light of this, the existence of cycles in the present framework can be regarded as a subproduct of myopic anticipations on future survival conditions.

Thus, the present model combines three features that are favorable to the occurence of cycles: (1) a dimension of the dynamic system larger than $1 ;(2)$ non-linearities; (3) myopic anticipations. Therefore, it should not surprise us that long-run cycles may exist in our model. Indeed it is only when $\eta$ tends towards 0 that cycles cannot arise. In that case, life expectancy is constant over time (i.e. $\pi_{t+1}=\pi_{t}=1$ ), so that the dimension of the dynamic system vanishes to 1 , while agents' expectations become perfect. ${ }^{37}$ Hence, although a

\footnotetext{
${ }^{36} \mathrm{On}$ the occurence of cycles in a larger class of OLG models, see the seminal work by Grandmont (1985), who shows that cycles may exist when the intertemporal elasticity of substitution is sufficiently smaller than unity.

${ }^{37}$ Indeed, agents make here a unique mistake concerning $\pi_{t+1}$, but anticipate $R_{t+1}$ perfectly.
} 
non-linearity remains in the capital accumulation process, cycles cannot arise under our standard assumptions on preferences and production.

Note, however, that even though one can rationalize the existence of longrun cycles in our model on the basis of three standard ingredients, the present framework brings nonetheless a significant contribution to the existing literature. Actually, the major virtue of the model is to complement the existing literature on economic cycles, by showing that the mere endogeneity of longevity, if coupled with myopic anticipations and a non-monotone survival function, may suffice to allow long-run cycles in output. Given that the imperfection of agents' anticipations on their survival prospects seems plausible, and that the non-monotonicity of the survival function is also reasonable, we are left here with the possible occurence of economic cycles despite standard assumptions on all the fundamentals of the economy (production and preferences). The demography suffices, under some conditions, to generate economic cycles.

The present paper complements also the literature on demographic cycles, which studies the determinants of the long-run dynamics of demographic variables. The pioneer contribution by Kuznets (1958) emphasized the existence of long swings (of length 15-25 years) in several demographic variables of the flow type (births, deaths, etc) over 1870-1955 in the U.S. ${ }^{38}$ According to Kuznets, such long fluctuations probably also prevailed in the U.S. during the nineteenth century, and constitute a phenomenon common to many societies and epochs.

Following Kuznets' pioneer works, a large attention was paid to fertility cycles, in particular by Easterlin $(1962,1973)$, who formulated what is now called the "Easterlin Hypothesis": the economic and social misfortunes of a birth cohort (including its fertility rate) depend on the cohort's relative size (approximated by the crude birth rate surrounding the cohort's birth). That hypothesis has as a corrolary that baby booms and baby busts must repeat perpetually over time. ${ }^{39}$ Theorists have tried to show that the Easterlin Hypothesis is compatible with OLG models with endogenous fertility. For instance, Benhabib and Nishimura (1989) showed, on the basis of an economy à la Barro and Becker (1989), that the fertility rate may well fluctuate endogenously. ${ }^{40}$

The cyclical nature of mortality has received, in comparison to fertility fluctuations, less attention. Actually, little attention has been paid, until the recent years, on mortality fluctuations. ${ }^{41}$ On the contrary, longevity has been rather used in a very different manner. In a well-known paper, Aiyagari (1989) showed that, provided the length of life of agents - taken here as exogenous - is sufficiently large, economic cycles must necessarily disappear when agents discount the future positively. ${ }^{42}$ At first glance, Aiyagari's result seems to be at odds with the present work: it takes longevity variations not at the origin of economic cycles, but at the origin of the disappearance of economic cycles. However, Aiyagari's results do not contradict the findings of the present study, since the latter takes longevity as endogenously rather than exogenously determined, and, as such, is concerned with longer time periods than Aiyagari's study. Therefore the

\footnotetext{
${ }^{38}$ Kuznets's (1965) analysis aimed at linking demographic and economic cycles.

${ }^{39}$ See Jeon and Shields (2005) on the validation of the Easterlin Hypothesis in the OECD.

${ }^{40}$ Note that the economic analysis of fluctuations in fertility is not restricted to the human kind. See Rosen et al (1994) on the fluctuations of the U.S. beef cattle stock.

${ }^{41}$ A recent exception is Ruhm (2000) in a short-run context.

${ }^{42}$ That result holds independently from the level of the intertemporal elasticity of substitution, unlike in the two-period model studied in Grandmont (1985).
} 
aims and skope of those two studies are complementary. Nonetheless, several connections can be made between Aiyagari's result and the present findings.

First of all, both papers emphasize the crucial role of discounting as far as the existence of cycles is concerned. Aiyagari showed that, provided agents discount the future positively, cycles must disappear when agents are sufficiently long-lived. In the present study, we also emphasize how the degree of impatience influences the occurence of cycles. As shown in Section 3, cycles cannot occur in societies of impatient agents, but only in societies whose members are sufficiently patient. Moreover, besides a common emphasis on time preferences, the present study shares also with Aiyagari (1989) a concern for the comparison of a human's lifecycle and a society's cycle. Aiyagari's result laid particular emphasis on a key issue in cycle analysis: the issue of the length of the economic cycle in comparison with the length of agents' own lifecycle. ${ }^{43}$ The issue of the length of the period of a cycle is also central for the understanding the skope of the present study. While the lengthening of the human lifecycle in Aiyagari (1989) was sufficient to make the period of cycles vanish to zero, we have, in the present model, that finite-lived agents can, by their unique savings decision based on their limited life horizon, give rise to an economic and demographic cycle whose length may be larger than a human life. ${ }^{44}$ Therefore the present study, by endogenizing individual lifespan (i.e. the key parameter in Aiyagari's work), casts new light on the articulation of individual lifecycles and social cycles.

The skope of the present work is thus very different from a large part of the business cycles literature, which is concerned with much shorter cycles. Actually, the present work is much closer to papers on very long-run economic growth, such as Galor and Moav (2005), who examine the dynamics of life expectancy in a heterogeneous economy composed of agents with unequal genetic background and children costs (the latter being correlated with the former, as "stronger" children are also more expensive to produce). Galor and Moav show that, from very long-run perspective, life expectancy does not follow a monotone evolution, but can exhibit fluctuations due to changes in the composition of the population. More precisely, variations in the total population size can contribute to shift the evolutionary advantage from one group of agents to another, which explains why, in the aggregate, longevity is not necessarily increasing. The present paper complements Galor and Moav's model by showing that longevity fluctuations can arise without having to rely on a heterogeneous population of agents: even without heterogeneity, one can, under some conditions, have longevity cycles.

In sum, this model complements the literature on economic and demographic cycles in various ways. True, the occurence of cycles in our economy is based on standard ingredients: a high dimension dynamic system $(>1)$, non-linearities and myopia. But the present study complements the literature by showing how, despite standard preferences and production technology, and despite the absence of heterogeneity, the introduction of a more realistic survival function in a standard two-period OLG model can, under some conditions, generate longrun fluctuations in longevity and output. Moreover, this casts also new light on

\footnotetext{
${ }^{43}$ Sims (1986) had emphasized that the observed business cycles exhibit a much shorter period than the ones predicted by OLG models. Aiyagari's results can thus be interpreted as stating that, as the (exogenous) lifetime becomes sufficiently long with respect to periods of economic activity, business cycles disappear.

${ }^{44}$ For instance, on Figures $8 \mathrm{a}$ and $8 \mathrm{~b}$, a cycle lasts 7 periods, that is, 3.5 human lives of maximum length. This is definitely longer than a standard Kondratieff cycle (45-65 years).
} 
the relationship between the individual lifecycle and the occurrence of economic and demographic cycles at the aggregate level.

\section{Conclusions}

The large theoretical literature on the relation between economic growth and longevity gains assumes usually that survival is increasing monotonically with the level of (physical or human) capital, either directly or indirectly (e.g. through health spending). Nevertheless, that postulate has a counterintuitive corollary: survival must, in general, be correlated positively with consumption, whatever the consumption level is. That corollary is not compatible with the epidemiological literature showing that excess consumption leads to a larger mortality.

This paper developed a two-period OLG model where the probability of survival to the second period is non-monotone in consumption, and is increasing in consumption only as long as consumption lies below a healthy consumption level. The study of the existence, uniqueness and stability of steady-state equilibria revealed that the dynamics of output and longevity varies strongly with the structural parameters of the economy, which can lead to either an overconsumption or an underconsumption equilibrium. The stability of the equilibrium is not guaranteed: cycles may exist around long-run equilibria, in the sense that periods of economic growth and longevity improvement would be followed by periods of economic contraction and lower life expectancy, and so forth.

Long-run economic and demographic cycles exist only around overconsumption equilibria, and under particular conditions on the structural parameters of the economy. Cycles are more plausible in economies with a high sensitivity of life expectancy to consumption behaviour, and where, because of exogenous reasons, the healthy consumption level is lower. Cycles are also more likely the more productive and capital-intensive the economy is. Those features are all shared by advanced economies, so that the present findings do not allow us to exclude a priori the possibility of future economic and demographic cycles due to the nefast effect of overconsumption on survival conditions.

Those findings invite two directions for future research. First, while the possible occurence of cycles in our model was shown to be due to the conjunction of three ingredients that favor the emergence of cycles (i.e. a dimension of the dynamic system exceeding unity, the presence of non-linearities and myopic anticipations), one may want to go further in the examination of the conditions under which cycles can or cannot occur, by relaxing some of those postulates. Second, given that the present model relies on a fixed production technology and a fixed medical technology, our findings on the possible occurence of longrun cycles could hardly be tested empirically, since both the production and the medical technology have evolved strongly over time. Therefore, in order to be able to test our model, it would be first necessary to generalize it slightly, by allowing such progress. ${ }^{45}$ Hence much work remains to be done to develop a satisfactory picture of the evolution of longevity and output across centuries.

\footnotetext{
${ }^{45}$ Interestingly, those two sources of progress would play opposite roles in terms of cycle's occurence: technological progress on the production side would probably reinforce instability, whereas medical progress could counteract the nefast effect of overconsumption, and make cycles less likely.
} 


\section{References}

Adams, K., Schatzkin, A., Harris, T., Kipnis, V., Mouw, T., Ballard-Barbash, R., Hollenbeck, A. \& Leitzmann, M. (2006): "Overweight, obesity, and mortality in a large prospective cohort of persons 50 to 71 years old", The New England Journal of Medicine, 355 (8), pp. 763-778.

Aiyagari, R. (1989): "Can there be short-period deterministic cycles when people are long lived?", Quarterly Journal of Economics, 104(1), pp. 163-185.

Anand, S. \& M. Ravallion (1993): "Human development in poor countries: on the role of private income and public services", Journal of Economic Perspectives, 7, pp. 133-150.

Barro, R. \& Becker, G.S. (1989): "Fertility choice in a model of economic growth", Econometrica, 57, pp. 481-501.

Bender, R., Trautner, C., Spraul, M. \& Berger, M. (1998): "Assessment of excess mortality in obesity", American Journal of Epidemiology, 147, 1, pp. 42-47.

Benhabib, J. \& Nishimura, K. (1989): "Endogenous fluctuations in the Barro-Becker theory of fertility", in A. Weig and K. Zimmermann (eds.): Demographic Change and Economic Development, Springer-Verlag.

Bhattacharya, J. \& Qiao, X. (2005): "Public and private expenditures on health in a growth model", Iowa State University Working Paper, \#05020.

Blackburn, K. \& Cipriani, G.P. (2002): "A model of longevity, fertility and growth", Journal of Economic Dynamics and Control, 26, pp. 187-204.

Boucekkine, R., de la Croix, D. \& O. Licandro (2002): "Vintage human capital, demographic trends, and endogenous growth", Journal of Economic Theory, 104, pp. 340-375.

Boucekkine, R., de la Croix, D. \& O. Licandro (2003): "Early mortality declines at the dawn of modern growth", Scandinavian Journal of Economics, 105, pp. 401-418.

Boucekkine, R., Diene, B. \& T. Azomahou (2009): "A closer look at the relationship between life expectancy and economic growth", International Journal of Economic Theory, 5 (2), pp. 201-244.

Breeze, E., Clarke, R., Shipley, M., Marmot, M., \& Fletcher, A. (2005): "Cause-specific mortality in old age in relation to body mass index in middle age and in old age: follow-up of the Whitehall cohort of male civil servants", International Journal of Epidemiology, 35(1), pp. 169-178.

Cervellati, M. \& Sunde, U. (2005): "Human capital formation, life expectancy, and the process of development", American Economic Review, 95, pp. 1653-1672.

Chakraborty, S. (2004): "Endogenous lifetime and economic growth", Journal of Economic Theory, 116, pp. 119-137.

Chakraborty, S. \& Das, M. (2005): "Mortality, human capital and persistent inequality", Journal of Economic Growth, 10, pp. 159-192.

Cutler, D., Glaeser, E. \& Shapiro, J. (2003): "Why have Americans become more obese?", Journal of Economic Perspectives, 17(3), pp. 93-118.

Day, R.H. (1982): "Irregular growth cycles", American Economic Review, 72, pp 406-414.

de la Croix, D. \& Licandro, O. (1999): "Life expectancy and endogenous growth", Economics Letters, 65, pp. 255-263.

de la Croix, D. \& Licandro, O. (2007): "The Child is the Father of Man", CORE Discussion Paper 2007-72.

de la Croix, D. \& Michel, P. (2002): A Theory of Economic Growth. Dynamics and Policy in Overlapping Generations. Cambridge University Press, Cambridge.

Easterlin, R. (1962): The American Baby Boom In A Historical Perspective, National Bureau of Economic Research, UMI Publisher.

Easterlin, R. (1973): "Relative economic status and the American fertility swing", in E. Sheldon (ed.): Family Economic Behavior: Problems and Prospects, Philadelphia, Lippincott. 
Ehrlich, I. \& Lui, F. (1991): "Intergenerational trade, longevity and economic growth", Journal of Political Economy, 99, pp. 1029-1060.

Farmer, R. (1986): "Deficits and cycles", Journal of Economic Theory, 6, pp. 77-88.

Fogel, R. (1994): "Economic growth, population theory and physiology: the bearing of long-term processes on the making of economic policy", American Economic Review, 84(3), pp. 369-395.

Fogel, R. (2004): "Health, nutrition and economic growth", Economic Development and Cultural Change, 52(3), pp. 643-658.

Fontaine, K., Redden, D., Wang, C., Westfall, A. \& Allison, D. (2003): "Years of life lost due to obesity", The Journal of the American Medical Association, 289(2), pp. 187-193.

Galor, O., \& Moav, O. (2005): "Natural selection and the evolution of life expectancy", CEPR, Minerva Center for Economic Growth Paper 02-05.

Grandmont, J.M. (1985): "On endogenous competitive business cycles", Econometrica, 53(5), pp. 995-1045.

Jeon, Y. \& Shields, M. (2005): "The Easterlin Hypothesis in the recent experience of higher-income OECD countries: a panel-data approach", Journal of Population Economics, 18 (1), pp. 1-13.

Jouvet, P.A., Pestieau, P., \& Ponthiere, G., (2010): "Longevity and environmental quality in an OLG model", Journal of Economics, 100 (3), pp. 191-216.

Kelley, A. \& R. Schmidt (1994): "Population and income change: recent evidence", World Bank Discussion Paper 249, Washington D.C.: World Bank.

Kelley, A. \& R. Schmidt (1995): "Aggregate population and economic growth correlations: the role of components of demographic change", Demography, 332, pp. 543-555.

Lakdawalla, D. \& Philipson, T. (2002): "The growth of obesity and technological change: a theoretical and empirical examination", NBER Discussion Paper 8946.

Medio, A. \& Lines, M. (2001): Non Linear Dynamics: A Primer. Cambridge University Press.

OECD (2009): OECD Statistics, data retrived on 01/08/2009,

at http://stats.oecd.org/index

Philipson, T. \& Posner, R. (2008): "Is the obesity epidemic a public health problem? A decade of research on the economics of obesity", NBER Discussion Paper 14010.

Ponthiere, G. (2010): "Unequal longevities and lifestyles transmission", Journal of Public Economic Theory, 12 (1), pp. 93-126.

Preston, S. H. (1975): "The changing relation between mortality and level of economic development", Population Studies, 29 (2), pp. 231-248.

Preston, S. H. (1980): "Causes and consequences of mortality declines in less developed countries during the twentieth century", in R. A. Easterlin (ed.): Population and Economic Changes in Developing Countries, Chicago, IL, Chicago Press.

Pritchett, L. \& L. Summers (1996): "Wealthier is healthier", Journal of Human Resources, 31 (4), pp. 841-868.

Rosen, S., Murphy, K. \& Scheinkman, J. (1994): "Cattle cycles", Journal of Political Economy, 102(3), pp. 468-492.

Ruhm, C. (2000): "Are recessions good for your health?", Quarterly Journal of Economics, 115(2), pp. 617-650.

Samuelson, P.A. (1939): "Interactions between the multiplier analysis and the principle of acceleration", Review of Economics and Statistics, 21, pp. 75-78.

Sims, C.A. (1986): "Comment", in H. Sonnenschein (ed.): Models of Economic Dynamics, Springer-Verlag: New-York, NY.

Solomon, C. \& Manson, J. (1997): "Obesity and mortality: a review of the epidemiological data", The American Journal of Clinical Nutrition, 66(4), pp. 1044-1050. 
Solow, R. (1956): "A contribution to the theory of economic growth", Quarterly Journal of Economics, 70(1), pp. 65-94.

World Health Organization Statistical Information System - WHOSIS (2009), data retrived on 01/09/2009, at http://apps.who.int/whosis/data/Search

Zhang, J., Zhang, J., \& Leung, M.C. (2006): "Health investment, saving, and public policy", Canadian Journal of Economics, 39, 1, pp. 68-93.

\section{Appendix}

\subsection{Proposition 1}

The existence of a steady-state equilibrium can be explored as the issue of the intersection of the $k k$ locus and the $\pi \pi$ locus in the two-dimensional space $\left(\pi_{t}, k_{t}\right)$. For that purpose, let us first derive the basic properties of those two loci.

We know that $G(0)=0$ and that, under $k_{t}=0, \pi_{t}$ is maintained constant at a level $\bar{\pi}$.

The first-order derivative of $G($.$) is$

$$
G^{\prime}\left(\pi_{t}\right)=\frac{1}{1-\alpha}[A(1-\alpha) \beta]^{\frac{1}{1-\alpha}} \pi_{t}^{\frac{1}{1-\alpha}-1}\left[\frac{1}{1+\beta \pi_{t}}\right]^{\frac{2-\alpha}{1-\alpha}}>0
$$

Regarding $H_{1}\left(\pi_{t}\right)$, we have, in the general case where $1 / \alpha$ is not an even integer:

$$
\lim _{\pi_{t} \rightarrow \bar{\pi}} H_{1}\left(\pi_{t}\right)=\left[(0) \frac{1}{A(1-\alpha)}\right]^{\frac{1}{\alpha}}=0
$$

In the special case when $1 / \alpha$ is an even integer, we have:

$$
\lim _{\pi_{t} \rightarrow 0} H_{1}\left(\pi_{t}\right)=\left[\left(c^{*}-\left(\frac{1}{\eta}\right)^{1 / 2}\left(\frac{1}{0}\right)^{1 / 2}\right) \frac{1}{A(1-\alpha)}\right]^{\frac{1}{\alpha}}=+\infty
$$

Moreover

$$
\begin{aligned}
H_{1}^{\prime}\left(\pi_{t}\right)= & \frac{1}{\alpha}\left[\left(c^{*}-\left(\frac{1}{\eta}\right)^{1 / 2}\left(\frac{1-\pi_{t}}{\pi_{t}}\right)^{1 / 2}\right) \frac{\left(1+\beta \pi_{t}\right)}{A(1-\alpha)}\right]^{\frac{1}{\alpha}-1} \\
& {\left[\left(\frac{1}{\eta}\right)^{1 / 2}\left(\frac{1}{2} \frac{\frac{1}{\pi_{t}}-\beta}{\pi^{1 / 2}(1-\pi)^{1 / 2} A(1-\alpha)}\right)+c^{*} \frac{\beta}{A(1-\alpha)}\right] }
\end{aligned}
$$

If $1 / \alpha$ is not an even integer, two cases may arise. First case: $(1 / \alpha)-1$ is not an even integer. In that case, the first factor is negative for low levels of $\pi_{t}<\bar{\pi}=\frac{1}{1+\eta c^{* 2}}$, but positive for $\pi_{t}>\bar{\pi}$. Given that the second factor is always positive, we have $H_{1}^{\prime}\left(\pi_{t}\right)<0$ for $\pi_{t}<\bar{\pi}$ and $H_{1}^{\prime}\left(\pi_{t}\right)>0$ for $\pi_{t}>\bar{\pi}$. At $\pi_{t}=\bar{\pi}$, we have $H_{1}^{\prime}\left(\pi_{t}\right)=0$. Second case: $(1 / \alpha)-1$ is an even integer. In that case, the first factor is always positive, whatever $\pi_{t}$ is. Given that the second factor is always positive (as $\beta \leq 1 \leq \frac{1}{\pi_{t}}$ ), we have thus $H_{1}^{\prime}\left(\pi_{t}\right)>0$.

However, if $1 / \alpha$ is an even integer, $(1 / \alpha)-1$ is an odd integer, so that the first factor is negative for low levels of $\pi_{t}<\bar{\pi}=\frac{1}{1+\eta c^{* 2}}$, but positive for $\pi_{t}>\bar{\pi}$. 
Given that the second factor is always positive, we have $H_{1}^{\prime}\left(\pi_{t}\right)<0$ for $\pi_{t}<\bar{\pi}$ and $H_{1}^{\prime}\left(\pi_{t}\right)>0$ for $\pi_{t}>\bar{\pi}$. At $\pi_{t}=\bar{\pi}$, we have $H_{1}^{\prime}\left(\pi_{t}\right)=0$.

Note also that, when $1 / \alpha$ is not an even integer,

$$
\begin{aligned}
\lim _{\pi_{t} \rightarrow \bar{\pi}} H_{1}^{\prime}\left(\pi_{t}\right) & =\frac{1}{\alpha}\left[(0) \frac{1}{A(1-\alpha)}\right]^{\frac{1}{\alpha}-1} \\
& {\left[\frac{1}{2}\left(\frac{1}{\bar{\pi}}\right)^{-1 / 2}\left(\frac{1}{\eta}\right)^{1 / 2} \frac{1}{\bar{\pi}}\left(\frac{1}{A(1-\alpha)}\right)+\left(c^{*}-\left(\frac{1}{\eta}\right)^{1 / 2}\left(\frac{1}{\bar{\pi}}\right)^{1 / 2}\right) \frac{\beta}{A(1-\alpha)}\right] } \\
= & 0
\end{aligned}
$$

In the special case where $1 / \alpha$ is an even integer,

$$
\begin{aligned}
\lim _{\pi_{t} \rightarrow 0} H_{1}^{\prime}\left(\pi_{t}\right)= & \frac{1}{\alpha}\left[\left(c^{*}-\left(\frac{1}{\eta}\right)^{1 / 2}\left(\frac{1}{0}\right)^{1 / 2}\right) \frac{1}{A(1-\alpha)}\right]^{\frac{1}{\alpha}-1} \\
& {\left[\frac{1}{2}\left(\frac{1}{0}\right)^{-1 / 2}\left(\frac{1}{\eta}\right)^{1 / 2} \frac{1}{0}\left(\frac{1}{A(1-\alpha)}\right)+\left(c^{*}-\left(\frac{1}{\eta}\right)^{1 / 2}\left(\frac{1}{0}\right)^{1 / 2}\right) \frac{\beta}{A(1-\alpha)}\right] } \\
= & -\infty
\end{aligned}
$$

and

$$
\begin{aligned}
\lim _{\pi_{t} \rightarrow 1} H_{1}^{\prime}\left(\pi_{t}\right) & =\frac{1}{\alpha}\left[c^{*} \frac{(1+\beta)}{A(1-\alpha)}\right]^{\frac{1}{\alpha}-1}\left[\frac{1}{2}\left(\frac{0}{1}\right)^{-1 / 2}\left(\frac{1}{\eta}\right)^{1 / 2}\left(\frac{1+\beta}{A(1-\alpha)}\right)+c^{*} \frac{\beta}{A(1-\alpha)}\right] \\
& =+\infty
\end{aligned}
$$

Regarding $H_{2}(\pi)$, we have

$$
\lim _{\pi_{t} \rightarrow 0} H_{2}\left(\pi_{t}\right)=\left[\left(c^{*}+\left(\frac{1}{\eta}\right)^{1 / 2}\left(\frac{1}{0}\right)^{1 / 2}\right) \frac{1}{A(1-\alpha)}\right]^{\frac{1}{\alpha}}
$$

Moreover,

$$
\begin{aligned}
H_{2}^{\prime}\left(\pi_{t}\right)= & \frac{1}{\alpha}\left[\left(c^{*}+\left(\frac{1}{\eta}\right)^{1 / 2}\left(\frac{1-\pi_{t}}{\pi_{t}}\right)^{1 / 2}\right) \frac{\left(1+\beta \pi_{t}\right)}{A(1-\alpha)}\right]^{\frac{1}{\alpha}-1} \\
& {\left[\left(\frac{1}{\eta}\right)^{1 / 2}\left(\frac{-\frac{1}{2} \pi_{t}^{1 / 2}\left(1+\beta \pi_{t}\right)+\beta\left(1-\pi_{t}\right)}{\pi_{t}^{2}\left(1-\pi_{t}\right)^{1 / 2} A(1-\alpha)}\right)+c^{*} \frac{\beta}{A(1-\alpha)}\right] }
\end{aligned}
$$

The first factor is always positive, whatever $\pi_{t}$ is. But the second factor can be positive or negative, depending on the level of $\pi_{t}$. Actually, if $\pi_{t}<\bar{\pi}=\frac{1}{1+\eta c^{* 2}}$, we always have $H_{2}^{\prime}\left(\pi_{t}\right)<0$, but this may not be the case for $\pi_{t}>\bar{\pi}$.

Note also that:

$$
\begin{aligned}
\lim _{\pi_{t} \rightarrow 0} H_{2}^{\prime}\left(\pi_{t}\right)= & \frac{1}{\alpha}\left[\left(c^{*}+\left(\frac{1}{\eta}\right)^{1 / 2}\left(\frac{1-0}{0}\right)^{1 / 2}\right) \frac{1}{A(1-\alpha)}\right]^{\frac{1}{\alpha}-1} \\
& {\left[\left(\frac{1}{\eta}\right)^{1 / 2}\left(\frac{-\frac{1}{2} 0_{t}^{1 / 2}+\beta}{0 A(1-\alpha)}\right)+c^{*} \frac{\beta}{A(1-\alpha)}\right] } \\
= & +\infty
\end{aligned}
$$


and

$$
\begin{aligned}
\lim _{\pi_{t} \rightarrow 1} H_{2}^{\prime}\left(\pi_{t}\right)= & \frac{1}{\alpha}\left[\left(c^{*}+\left(\frac{1}{\eta}\right)^{1 / 2}(0)^{1 / 2}\right) \frac{(1+\beta)}{A(1-\alpha)}\right]^{\frac{1}{\alpha}-1} \\
& {\left[\frac{1}{2}(0)^{-1 / 2}\left(\frac{1}{\eta}\right)^{1 / 2} \frac{-1}{1}\left(\frac{1+\beta}{A(1-\alpha)}\right)+\left(c^{*}+\left(\frac{1}{\eta}\right)^{1 / 2}\left(\frac{0}{1}\right)^{1 / 2}\right) \frac{\beta}{A(1-\alpha)}\right] } \\
= & -\infty
\end{aligned}
$$

Let us now consider the existence problem on a case-by-case basis. For that purpose, we shall use the following notations: $G(1)=\left[\frac{\beta A(1-\alpha)}{1+\beta}\right]^{\frac{1}{1-\alpha}}$ is denoted by $k^{*}$, while $H_{1}(1)=H_{2}(1)=\left[c^{*} \frac{1+\beta}{A(1-\alpha)}\right]^{\frac{1}{\alpha}}$ is denoted by $\Omega$.

First of all, given that a zero capital level maintains itself over time for any level of $\pi_{t}$, and that a zero level of capital makes the probability of survival constant at a minimum level $\bar{\pi}$, it follows that $(\bar{\pi}, 0)$ is a stationary equilibrium, at which underconsumption prevails.

In order to treat the existence of other steady-states, let us distinguish between the different cases.

Regarding case (1), where $\frac{1}{\alpha}$ is not an even integer and where $k^{*}<\Omega$, there must exist at least one steady-state with $\pi>\bar{\pi}$ and $k>0$. Indeed, the $\pi \pi$ locus lies, at $\pi=1$, above the $k k$ locus: $H_{1}(1)=H_{2}(1)>G(1)$. However, given that $H_{1}(\bar{\pi})=0<G(\bar{\pi})$, it follows, by continuity of $H_{1}($.$) and G($.$) , that H_{1}($.$) must$ intersect $G($.$) at least once. That underconsumption equilibrium is denoted by$ $\left(\pi_{1}, k_{1}\right)$ in Proposition 1.

Regarding case (2), where $\frac{1}{\alpha}$ is not an even integer and where $k^{*}>\Omega$, there must also exist at least one steady-state with $\pi>0$ and $k>0$. Indeed, the $\pi \pi$ locus lies, at $\pi=1$, below the $k k$ locus: $H_{1}(1)=H_{2}(1)<G(1)$. However, given that $G($.$) tends to zero as \pi_{t}$ tends to 0 , whereas $H_{2}($.$) tends to infinity as \pi_{t}$ tends to $0, G\left(\pi_{t}\right)$ and $H_{2}\left(\pi_{t}\right)$ must intersect somewhere. That overconsumption equilibrium is denoted by $\left(\pi_{2}, k_{2}\right)$ in Proposition 1 . Note that, as it is an overconsumption equilibrium, we know that $k_{2}>k^{*}$, but not whether $\pi_{2} \gtrless \bar{\pi}$.

Regarding case (3), where $\frac{1}{\alpha}$ is not an even integer and where $k^{*}=\Omega$, the $\pi \pi$ locus lies, at $\pi_{t}=1$, at exactly the same level as the $k k$ locus: $H_{1}(1)=$ $H_{2}(1)=G(1)$, so that $\left(1, k^{*}\right)$ is an equilibrium. There cannot be any other healthy consumption equilibrium, because a unique level of $k$ can yield $\pi=1$.

Regarding case (4), where $\frac{1}{\alpha}$ is an even integer and where $k^{*}<\Omega$, there must exist at least two steady-states with $\pi>0$ and $k>0$. Indeed, $H_{1}(1)=H_{2}(1)>$ $G(1)$. Furthermore, $H_{1}($.$) tends to 0$ when $\pi_{t}$ tends to $\bar{\pi}$, whereas $G($.$) tends$ to zero as $\pi_{t}$ tends to 0 , so that $G\left(\pi_{t}\right)$ and $H_{1}\left(\pi_{t}\right)$ must necessarily intersect somewhere, at an equilibrium denoted by $\left(\pi_{4}, k_{4}\right)$, at which $\pi_{4}>\bar{\pi}$ and $k_{4}>0$. That equilibrium is an underconsumption equilibrium. Moreover, under $\frac{1}{\alpha}$ an even integer, $H_{1}($.$) tends also to +\infty$ when $\pi_{t}$ tends to zero. Because of this, it must also be the case that $G\left(\pi_{t}\right)$ and $H_{1}\left(\pi_{t}\right)$ intersect at an equilibrium with $\pi<$ $\bar{\pi}$ and $k>0$. That equilibrium, denoted $\left(\pi_{3}, k_{3}\right)$, is also an underconsumption equilibrium, and must lie below $\left(\pi_{4}, k_{4}\right)$, as it must be at $\pi_{3}<\bar{\pi}$, and as $G($. increasing in $\pi_{t}$. Thus we must have $\pi_{4}>\pi_{3}$ and $k_{4}>k_{3}$.

Regarding case (5), where $\frac{1}{\alpha}$ is an even integer and where $k^{*}>\Omega$, there must exist at least two steady-states with $\pi>0$ and $k>0$. Indeed, $H_{1}(1)=$ 
$H_{2}(1)<G(1)$. Furthermore, we know that $H_{2}($.$) tends to +\infty$ when $\pi_{t}$ tends to zero, whereas $G($.$) tends to zero as \pi_{t}$ tends to 0 , so that $G\left(\pi_{t}\right)$ and $H_{2}\left(\pi_{t}\right)$ must necessarily intersect somewhere, at $\left(\pi_{6}, k_{6}\right)$, which is an overconsumption equilibrium, at which $k_{6}>k^{*}$, and $\pi_{6} \lessgtr \bar{\pi}$. Moreover, under $\frac{1}{\alpha}$ an even integer, $H_{1}$ (.) tends to $+\infty$ when $\pi_{t}$ tends to zero. Because of this, it must be the case that $G\left(\pi_{t}\right)$ and $H_{1}\left(\pi_{t}\right)$ intersect somewhere else, at another equilibrium with $\pi>0$ and $k>0$. That equilibrium, denoted $\left(\pi_{5}, k_{5}\right)$, is an underconsumption equilibrium, and lies below $\left(\pi_{6}, k_{6}\right)$, as $H_{1}()<.H_{2}($.$) for any \pi_{t} \neq 1$ and $G($. increasing in $\pi_{t}$. Thus we must have $\pi_{6}>\pi_{5}$ and $k_{6}>k_{5}$.

Regarding case (6), where $\frac{1}{\alpha}$ is an even integer and where $k^{*}=\Omega$, we have $H_{1}(1)=H_{2}(1)=G(1)$, so that $\left(1, k^{*}\right)$ is an equilibrium. Moreover, under $\frac{1}{\alpha}$ even integer, $H_{1}($.$) tends to +\infty$ when $\pi_{t}$ tends to zero. Because of this, it must also be the case that $G\left(\pi_{t}\right)$ and $H_{1}\left(\pi_{t}\right)$ intersect somewhere else, at an equilibrium with $\pi<\bar{\pi}$ and $k<k^{*}$. That equilibrium, denoted $\left(\pi_{7}, k_{7}\right)$, is an underconsumption equilibrium, and must lie below $\left(1, k^{*}\right)$.

Finally, it should be stressed that the above statements concern the necessary existence of some minimal number of stationary equilibria. However, the ambiguous signs of the second-order derivatives of both $G\left(\pi_{t}\right), H_{1}\left(\pi_{t}\right)$ and $H_{2}\left(\pi_{t}\right)$ do not allow us to draw conclusions regarding the possible existence of a larger number of steady-state equilibria. Indeed, the second-order derivative of the $k k$ locus is

$$
G^{\prime \prime}\left(\pi_{t}\right)=\frac{1}{1-\alpha}[A(1-\alpha) \beta]^{\frac{1}{1-\alpha}}\left[\frac{1}{1+\beta \pi_{t}}\right]^{\frac{2-\alpha}{1-\alpha}} \pi_{t}^{\frac{1}{1-\alpha}-2}\left[\frac{\alpha-2 \beta \pi_{t}(1-\alpha)}{(1-\alpha) 1+\beta \pi_{t}}\right]
$$

Thus, the $k k$ locus is convex when $\alpha>2 \beta \pi_{t}(1-\alpha)$ and concave for $\alpha<$ $2 \beta \pi_{t}(1-\alpha)$. Put it differently, we have $G^{\prime \prime}()>$.0 when $\pi_{t}<\tilde{\pi} \equiv \frac{\alpha}{2 \beta(1-\alpha)}$, and $G^{\prime \prime}()<$.0 when $\pi_{t}>\tilde{\pi} \equiv \frac{\alpha}{2 \beta(1-\alpha)}$.

Moreover, after simplifications, the second-order derivative of the low branch of the $\pi \pi$ locus is

$$
\begin{aligned}
H_{1}^{\prime \prime}\left(\pi_{t}\right)= & \frac{1}{\alpha}\left(\frac{1}{\alpha}-1\right)\left[\left(c^{*}-\left(\frac{1}{\eta}\right)^{1 / 2}\left(\frac{1-\pi_{t}}{\pi_{t}}\right)^{1 / 2}\right) \frac{\left(1+\beta \pi_{t}\right)}{A(1-\alpha)}\right]^{\frac{1}{\alpha}-2} \\
& {\left[\left(\frac{1}{\eta}\right)^{1 / 2}\left(\frac{1}{2} \frac{\frac{1}{\pi_{t}}-\beta}{\pi^{1 / 2}(1-\pi)^{1 / 2} A(1-\alpha)}+c^{*} \frac{\beta}{A(1-\alpha)}\right]^{2}\right.} \\
& +\frac{1}{\alpha}\left[\left(c^{*}-\left(\frac{1}{\eta}\right)^{1 / 2}\left(\frac{1-\pi_{t}}{\pi_{t}}\right)^{1 / 2}\right) \frac{\left(1+\beta \pi_{t}\right)}{A(1-\alpha)}\right]^{\frac{1}{\alpha}-1} \\
& {\left[\left(\frac{1}{\eta}\right)^{1 / 2} \frac{1}{2}\left(\frac{-\left(1-\pi_{t}\right)^{1 / 2}-\frac{1}{2}\left[\frac{\left(\frac{1}{\pi_{t}}-\beta\right)}{\pi_{t}^{1 / 2}\left(1-\pi_{t}\right)^{1 / 2}}\right]}{\left[\pi_{t}^{1 / 2}\left(1-\pi_{t}\right)^{1 / 2}\right]^{2} A(1-\alpha)}\right)\right] }
\end{aligned}
$$

If $1 / \alpha$ is not an even integer, so is $(1 / \alpha)-2$, so that the first term is positive if $\pi_{t}<\bar{\pi}$ and negative if $\pi_{t}>\bar{\pi}$. However, the second term is always negative. Hence, for $\pi_{t}>\bar{\pi}$, we always have $H_{1}^{\prime \prime}\left(\pi_{t}\right)<0$, that is, the convexity of the $\pi \pi$ locus in the positive orthan of the $\left(\pi_{t}, k_{t}\right)$ space. If $1 / \alpha$ is even, so is $(1 / \alpha)-2$, so that the first term is always positive. However, the second term is negative 
if $\pi_{t}<\bar{\pi}$ and positive if $\pi_{t}>\bar{\pi}$. Hence, for $\pi_{t}>\bar{\pi}$, we always have $H_{1}^{\prime \prime}\left(\pi_{t}\right)>0$, that is, the convexity of the $\pi \pi$ locus in its increasing part.

Finally, the second-order derivative of the high branch of the $\pi \pi$ locus is

$$
\begin{aligned}
H_{2}^{\prime \prime}\left(\pi_{t}\right)= & \frac{1}{\alpha}\left(\frac{1}{\alpha}-1\right)\left[\left(c^{*}+\left(\frac{1}{\eta}\right)^{1 / 2}\left(\frac{1-\pi_{t}}{\pi_{t}}\right)^{1 / 2}\right) \frac{\left(1+\beta \pi_{t}\right)}{A(1-\alpha)}\right]^{\frac{1}{\alpha}-2} \\
& {\left[\left(\frac{1}{\eta}\right)^{1 / 2}\left(\frac{-\frac{1}{2} \pi_{t}^{1 / 2}\left(1+\beta \pi_{t}\right)+\beta\left(1-\pi_{t}\right)}{\pi_{t}^{2}\left(1-\pi_{t}\right)^{1 / 2} A(1-\alpha)}\right)+c^{*} \frac{\beta}{A(1-\alpha)}\right]^{2} } \\
& +\frac{1}{\alpha}\left[\left(c^{*}+\left(\frac{1}{\eta}\right)^{1 / 2}\left(\frac{1-\pi_{t}}{\pi_{t}}\right)^{1 / 2}\right) \frac{\left(1+\beta \pi_{t}\right)}{A(1-\alpha)}\right]^{\frac{1}{\alpha}-1} \\
& {\left[\left(\frac{1}{\eta}\right)^{1 / 2}\left(\frac{-\frac{1}{4} \pi_{t}^{-1 / 2}\left(1+\beta \pi_{t}\right)-\beta \frac{1}{2} \pi_{t}^{1 / 2}-\beta+\frac{\frac{1}{2}\left(1+\beta \pi_{t}\right)\left(4-5 \pi_{t}\right)}{2 \pi_{t}^{1 / 2}\left(1-\pi_{t}\right)}-\frac{\beta\left(4-5 \pi_{t}\right)}{2 \pi_{t}}}{\pi_{t}^{2}\left(1-\pi_{t}\right)^{1 / 2} A(1-\alpha)}\right)\right] }
\end{aligned}
$$

The first term is unambiguously positive, as the second factor is at the power two. However, the sign of the second term is ambiguous, as the second factor may be either positive or negative.

It follows from the ambiguous signs of the second-order derivatives that the uniqueness of intersections of those loci in different areas of the $\left(\pi_{t}, k_{t}\right)$ space cannot be taken for granted.

\subsection{Proposition 2}

Let us now study formally the stability of the long-run equilibria identified in Section 3. First of all, let us notice that the present dynamic system is non linear. Indeed, the transition functions for capital per worker and the survival probability are:

$$
\begin{aligned}
k_{t+1} & =\frac{\beta \pi_{t}}{1+\beta \pi_{t}} A(1-\alpha) k_{t}^{\alpha} \\
\pi_{t+1} & =\frac{1}{1+\eta\left(c^{*}-\left(\frac{1}{1+\beta \pi_{t}} A(1-\alpha) k_{t}^{\alpha}\right)\right)^{2}}
\end{aligned}
$$

The non linearity of the system of dynamic equations implies that the conventional analysis of the Jacobian matrix (composed of the first-order derivatives of dynamic equations with respect to state variables) can only inform us on the stability of equilibria provided these are hyperbolic.

Actually, if a fixed point is hyperbolic, the Hartman-Grobman theorem states that the stability of the linearized system (or its non stability) implies the local stability of the non-linear system (or its non stability) (see Medio and Lines, 2001). However, if a fixed point is not hyperbolic, then the analysis of the linearized system does not allow us to draw conclusions on the local stability of the non linear system.

As stated by Medio and Lines (2001), fixed points in discrete-time systems are hyperbolic if none of the eigenvalues of the Jacobian matrix, evaluated at the equilibrium, is equal to 1 in modulo. 
Thus, to discuss the hyperbolicity of the equilibria characterized in Section 3, let us first compute the Jacobian matrix and study its properties. The Jacobian matrix is

$$
J \equiv\left(\begin{array}{ll}
\frac{\partial k_{t+1}}{\partial k_{t}} & \frac{\partial k_{t+1}}{\partial \pi_{t}} \\
\frac{\partial \pi_{t+1}}{\partial k_{t}} & \frac{\partial \pi_{t+1}}{\partial \pi_{t}}
\end{array}\right)
$$

where the entries are estimated at the equilibrium $(\pi, k)$.

The entries of the Jacobian matrix are:

$$
\begin{aligned}
\frac{\partial k_{t+1}}{\partial k_{t}} & =\alpha<1 \\
\frac{\partial k_{t+1}}{\partial \pi_{t}} & =\frac{\beta c}{1+\beta \pi}>0 \\
\frac{\partial \pi_{t+1}}{\partial k_{t}} & =\frac{2 \alpha \eta\left(c^{*}-c\right)}{\beta \pi\left[1+\eta\left(c^{*}-c\right)^{2}\right]^{2}} \\
\frac{\partial \pi_{t+1}}{\partial \pi_{t}} & =\frac{-2 \eta\left(c^{*}-c\right)\left(\frac{\beta c}{1+\beta \pi}\right)}{\left[1+\eta\left(c^{*}-c\right)^{2}\right]^{2}}
\end{aligned}
$$

where $c$ denotes the equilibrium consumption, i.e. $\left(\frac{1}{1+\beta \pi}\right)^{\frac{1}{1-\alpha}}[A(1-\alpha)]^{\frac{1}{1-\alpha}}[\beta \pi]^{\frac{\alpha}{1-\alpha}}$.

Hence the determinant of the Jacobian matrix is

$$
\operatorname{det}(J)=-2 \alpha \eta\left(c^{*}-c\right) \frac{\left(\frac{\beta c}{1+\beta \pi}\right)\left(1+\frac{1}{\beta \pi}\right)}{\left[1+\eta\left(c^{*}-c\right)^{2}\right]^{2}}
$$

The trace of the Jacobian matrix is

$$
\operatorname{tr}(J)=\alpha-\frac{2 \eta\left(c^{*}-c\right)\left(\frac{\beta c}{1+\beta \pi}\right)}{\left[1+\eta\left(c^{*}-c\right)^{2}\right]^{2}}
$$

As stated in Medio and Lines (2001, p. 52), the conditions that are necessary and sufficient having two eigenvalues of the Jacobian matrix smaller than 1 in modulo can be written as

$$
\begin{aligned}
1+\operatorname{tr}(J)-\operatorname{det}(J) & >0 \\
1-\operatorname{tr}(J)+\operatorname{det}(J) & >0 \\
1-\operatorname{det}(J) & >0
\end{aligned}
$$

Substituting for the trace and the determinant of the Jacobian matrix yields:

$$
\begin{gathered}
(1+\alpha)\left[1+\eta\left(c^{*}-c\right)^{2}\right]^{2}-\left[2 \eta\left(c^{*}-c\right)\left(\frac{\beta c}{1+\beta \pi}\right)\right]\left[1+\alpha \frac{1+\beta \pi}{\beta \pi}\right]>0 \\
(1-\alpha)\left[1+\eta\left(c^{*}-c\right)^{2}\right]^{2}+\left[2 \eta\left(c^{*}-c\right)\left(\frac{\beta c}{1+\beta \pi}\right)\right]\left[1-\alpha \frac{1+\beta \pi}{\beta \pi}\right]>0 \\
{\left[1+\eta\left(c^{*}-c\right)^{2}\right]^{2}+2 \alpha \eta\left(c^{*}-c\right) \frac{c}{\pi}>0}
\end{gathered}
$$


Note that those conditions are necessary and sufficient for the hyperbolicity and the stability of a steady-state equilibrium $(\pi, k)$. Note that, as far as the equilibrium $(\bar{\pi}, 0)$ are concerned, it is easy to see that those conditions are always satisfied. Indeed, under a zero equilibrium capital level, $\operatorname{tr}(J)$ equals $\alpha$ and $\operatorname{det}(J)$ equals 0 . Finally, substituting for $\pi$ in the three expressions above yields the conditions of Proposition 2.

\subsection{Proposition 3}

Having stated the general conditions for stability, let us look, on a case-by-case basis, at the various possible scenarios for the dynamics. For that purpose, let us notice, following Medio and Lines (2001), that the characteristic equation is

$$
\lambda^{2}-\operatorname{tr}(J) \lambda+\operatorname{det}(J)=0
$$

Thus the eigenvalues can be written as

$$
\begin{aligned}
\lambda_{1,2} & =\frac{1}{2}\left(\operatorname{tr}(J) \pm \sqrt[2]{[\operatorname{tr}(J)]^{2}-4 \operatorname{det}(J)}\right) \\
& =\frac{1}{2}(\operatorname{tr}(J) \pm \sqrt[2]{\Delta})
\end{aligned}
$$

The term $\Delta$ is equal to

$$
\begin{aligned}
\Delta & \equiv\left[\frac{\alpha\left[1+\eta\left(c^{*}-c\right)^{2}\right]^{2}-2 \eta\left(c^{*}-c\right)\left(c \frac{\beta}{1+\beta \pi}\right)}{\left[1+\eta\left(c^{*}-c\right)^{2}\right]^{2}}\right]^{2}+8 \frac{\alpha \eta\left(c^{*}-c\right)\left(\frac{\beta c}{1+\beta \pi}\right)\left(1+\frac{1}{\beta \pi}\right)}{\left[1+\eta\left(c^{*}-c\right)^{2}\right]^{2}} \\
& >0 \text { if } c^{*} \geq c \\
& \lessgtr 0 \text { if } c^{*}<c
\end{aligned}
$$

Let us now distinguish between the different cases.

Case (1) Under case (1), we know that the capital per worker at the equilibrium is lower than the one maximizing $\pi$. Hence, it must be the case that we are in an equilibrium of underconsumption: $c<c^{*}$. Thus we have $\frac{\partial \pi_{t+1}}{\partial k_{t}}>0$ and $\frac{\partial \pi_{t+1}}{\partial \pi_{t}}<0$. Therefore, $\operatorname{det}(J)$ is negative, indicating that the eigenvalues of the Jacobian matrix have opposite signs. The trace has an ambiguous sign, as the second term is negative. Moreover, we have $\Delta>0$. Hence three cases can arise:

- $\left|\lambda_{1}\right|<1,\left|\lambda_{2}\right|<1$, we have a stable equilibrium. Given that $\operatorname{det}(J)<0$, we have improper oscillations, but there is a non-monotone convergence.

- $\left|\lambda_{1}\right|>1,\left|\lambda_{2}\right|>1$, we have an unstable equilibrium, with improper oscillations. There is no convergence.

- $\left|\lambda_{1}\right|>1,\left|\lambda_{2}\right|<1$, the equilibrium is a saddle point.

Hence, the stability of the equilibrium $\left(\pi_{1}, k_{1}\right)$ depends on the one of those three cases under which we fall. 
Case (2) Under case (2), we have $c>c^{*}$. Hence, we have $\frac{\partial \pi_{t+1}}{\partial k_{t}}<0$ and $\frac{\partial \pi_{t+1}}{\partial \pi_{t}}>0$. Thus, in that case, $\operatorname{det}(J)$ is positive, and the trace is positive. Hence the two eigenvalues are positive. However, we do not know the sign of $\Delta$.

If $\Delta>0$, three cases can arise:

- $\left|\lambda_{1}\right|<1,\left|\lambda_{2}\right|<1$, we have a stable equilibrium. Given that $\operatorname{det}(J)>0$, we have a monotone convergence.

- $\left|\lambda_{1}\right|>1,\left|\lambda_{2}\right|>1$, we have an unstable equilibrium, with a monotone divergence.

- $\left|\lambda_{1}\right|>1,\left|\lambda_{2}\right|<1$, the equilibrium is a saddle point.

If $\Delta<0$, the two eigenvalues are a complex conjugate pair $\lambda_{1}, \lambda_{2}=\sigma \pm \theta i$, and the solutions are sequences of points situated on spirals. Three cases can arise:

- If $\sqrt[2]{\operatorname{det}(J)}<1$, the solutions converge to equilibrium point, which is a stable equilibrium.

- If $\sqrt[2]{\operatorname{det}(J)}>1$, the solutions diverge and the equilibrium point is unstable.

- If $\sqrt[2]{\operatorname{det}(J)}=1$, the eigenvalues lie exactly on the unit circle. Hence the equilibrium point is unstable, as we have a cycle around it.

If $\Delta=0$, there is a repeated real eigenvalue $\lambda=\operatorname{Tr}(J) / 2$. Hence the eigenvalues are

$$
\frac{\alpha-\frac{2 \eta\left(c^{*}-c\right)\left(c \frac{\beta}{1+\beta \pi}\right)}{\left[1+\eta\left(c^{*}-c\right)^{2}\right]^{2}}}{2}
$$

Given that we have here $\left(c^{*}-c\right)<0$, this expression is always positive. If this is smaller than 1 , we have a convergence towards the steady-state. If it is equal or larger than 1 , we have a divergence and the equilibrium is unstable.

Note that if we impose the condition of Proposition 3:

$$
\Lambda \equiv \alpha-\frac{2 \eta\left(c^{*}-c\right)\left(c \frac{\beta}{1+\beta \pi}\right)}{\left[1+\eta\left(c^{*}-c\right)^{2}\right]^{2}}<1
$$

it is easy to see that the three conditions of Proposition 2 become:

$$
\begin{aligned}
1+\Lambda+\alpha[\Lambda-\alpha] & \left(1+\frac{1}{\beta \pi}\right)>0 \\
1-\Lambda+\alpha[\Lambda-\alpha]\left(1+\frac{1}{\beta \pi}\right) & >0 \\
1-\alpha[\Lambda-\alpha]\left(1+\frac{1}{\beta \pi}\right) & >0
\end{aligned}
$$

Under case (2), we have $\Lambda>0$. Hence, if $\Lambda<1$, the three conditions above are necessarily satisfied. Hence the above condition suffices to guarantee the local stability of the equilibrium $\left(\pi_{2}, k_{2}\right)$. However, the precise form of the convergence - monotone or not - depends on whether $\Delta \geq 0$ or $\Delta<0$. In the former case, we will observe a monotone convergence, whereas in the latter, there will be a spiral converging towards the equilibrium. 
Case (3) Under case (3), we have $c=c^{*}$, so that $\Delta>0$. We also have $\operatorname{det}(J)=0$ and $\operatorname{tr}(J)=\alpha$, from which it follows that the two eigenvalues must be 0 and $\alpha$. As a consequence, we fall under the case where $\left|\lambda_{1}\right|<1$ and $\left|\lambda_{2}\right|<1$, so that we have a stable equilibrium. We also have monotone convergence towards that equilibrium. Thus $\left(1, k^{*}\right)$ is locally stable.

Case (4) Under case (4), we have, at each equilibrium, $c<c^{*}$. Thus $\operatorname{det}(J)$ is negative, indicating that the eigenvalues of $J$ have opposite signs. The trace has an ambiguous sign. One can apply the same procedure as in case (1) to determine the stability of equilibria $\left(\pi_{3}, k_{3}\right)$ and $\left(\pi_{4}, k_{4}\right)$.

Case (5) Under case (5), the low equilibrium $\left(\pi_{5}, k_{5}\right)$ is such that $c<c^{*}$. Hence $\operatorname{det}(J)$ is, at that equilibrium, negative, so that the eigen values are of opposite signs, and the trace is of an ambiguous sign. On the contrary, at the high equilibrium $\left(\pi_{6}, k_{6}\right)$, we have $c>c^{*}$. Hence, in that case, $\operatorname{det}(J)$ is positive, and the trace is positive. Hence the two eigen values are positive.

The analysis of local stability is the same as under case (2). Given that the two eigenvalues of the Jacobian matrix are positive, the condition of Proposition 3 guarantees the hyperbolicity of the equilibrium and its local stability. Indeed, under that condition, the two eigen values, which are positive, are strictly lower than 1 (as the trace, i.e. their sum, is lower than 1) and so hyperbolicity prevails. Moreover, the conditions of Proposition 2 are also satisfied.

Case (6) As in case (3), we have $c=c^{*}$, so that $\Delta>0$. We also have $\operatorname{det}(J)=0$ and $\operatorname{tr}(J)=\alpha$, from which it follows that the two eigenvalues must be 0 and $\alpha$. As a consequence, we fall under the case where $\left|\lambda_{1}\right|<1$ and $\left|\lambda_{2}\right|<1$, so that we have a stable equilibrium. We also have monotone convergence towards that equilibrium. Thus $\left(1, k^{*}\right)$ is locally stable.

At $\left(\pi_{7}, k_{7}\right)$, we have $c<c^{*}$. Thus $\operatorname{det}(J)$ is negative, indicating that the eigen values of the Jacobian matrix have opposite signs. The trace has an ambiguous sign. One can apply the same procedure as in case (1) to determine the stability of the equilibrium $\left(\pi_{7}, k_{7}\right)$.

\subsection{Proposition 4}

Note first that, as far as the equilibria $(\bar{\pi}, 0)$ are concerned, the stability conditions are always satisfied, so that there can be no cycle in those cases.

Actually, in order to have long-run cycles, we need, as shown above, $\Delta<0$, that is, that the eigenvalues of the Jacobian matrix are complex.

Under cases (1) and (4), where the long-run equilibrium is an underconsumption equilibrium, we always have $\Delta>0$, so that cycles cannot occur around equilibria $\left(\pi_{1}, k_{1}\right),\left(\pi_{3}, k_{3}\right)$ and $\left(\pi_{4}, k_{4}\right)$.

Under cases (2) and (5), we can have $\Delta<0$. The first condition of Proposition 4 states the condition for $\Delta<0$. Indeed $\Delta<0$ if and only if

$$
\left[\frac{\alpha\left[1+\eta\left(c^{*}-c\right)^{2}\right]^{2}-2 \eta\left(c^{*}-c\right)\left(\frac{\beta c}{1+\beta \pi}\right)}{\left[1+\eta\left(c^{*}-c\right)^{2}\right]^{2}}\right]^{2}+8 \frac{\alpha \eta\left(c^{*}-c\right)\left(\frac{\beta c}{1+\beta \pi}\right)\left(1+\frac{1}{\beta \pi}\right)}{\left[1+\eta\left(c^{*}-c\right)^{2}\right]^{2}}<0
$$


Substituting for $\pi$ yields:

$$
\left[\frac{\alpha\left[1+\eta\left(c^{*}-c\right)^{2}\right]-\frac{2 \eta\left(c^{*}-c\right) \beta c}{1+\beta+\eta\left(c^{*}-c\right)^{2}}}{1+\eta\left(c^{*}-c\right)^{2}}\right]^{2}+8 \frac{\alpha \eta\left(c^{*}-c\right) c}{1+\eta\left(c^{*}-c\right)^{2}}<0
$$

This condition excludes the possibility of cycles around the low equilibrium of case (5), that is, around $\left(\pi_{5}, k_{5}\right)$, because that equilibrium is an underconsumption equilibrium, at which we necessarily have $\Delta>0$. But the condition $\Delta<0$ may be true at $\left(\pi_{2}, k_{2}\right)$ and $\left(\pi_{6}, k_{6}\right)$, which are overconsumption equilibria. The second condition of Proposition 4 is equivalent to $\sqrt[2]{\operatorname{det}(J)}=1$, as required for the existence of a cycle. Indeed, $\sqrt[2]{\operatorname{det}(J)}=1$ can be written as:

$$
\sqrt[2]{\frac{-2 \alpha \eta\left(c^{*}-c\right)\left(\frac{\beta c}{1+\beta \pi}\right)\left(1+\frac{1}{\beta \pi}\right)}{\left[1+\eta\left(c^{*}-c\right)^{2}\right]^{2}}}=1
$$

Substituting for $\pi$ yields:

$$
\sqrt[2]{\frac{-2 \alpha \eta\left(c^{*}-c\right) c}{1+\eta\left(c^{*}-c\right)^{2}}}=1
$$

Here again, that condition may be satisfied at equilibria $\left(\pi_{2}, k_{2}\right)$ and $\left(\pi_{6}, k_{6}\right)$, depending on the structural parameters of the economy. Hence taken together, the 2 conditions of Proposition 4 guarantee the existence of long-run cycles around $\left(\pi_{2}, k_{2}\right)$ and $\left(\pi_{6}, k_{6}\right)$.

Under cases (3) and (6), we have $\Delta>0$, so that cycles cannot occur around equilibria $\left(1, k^{*}\right)$ and $\left(\pi_{7}, k_{7}\right)$. 